\title{
MinerLSD: efficient mining of local patterns on attributed networks
}

\author{
Martin Atzmueller ${ }^{1,2^{*}}$ (D) , Henry Soldano ${ }^{3,4}$, Guillaume Santini ${ }^{3}$ and Dominique Bouthinon ${ }^{3}$
}

\author{
${ }^{*}$ Correspondence: \\ m.atzmuller@uvt.nl \\ ${ }^{1}$ Tilburg University, Department of \\ Cognitive Science and Artificial \\ Intelligence, Warandelaan 2, 5037 \\ AB Tilburg, The Netherlands \\ ${ }^{2}$ Université Sorbonne Paris Cité, \\ Paris, France \\ Full list of author information is \\ available at the end of the article
}

\begin{abstract}
Local pattern mining on attributed networks is an important and interesting research area combining ideas from network analysis and data mining. In particular, local patterns on attributed networks allow both the characterization in terms of their structural (topological) as well as compositional features. In this paper, we present MinerLSD, a method for efficient local pattern mining on attributed networks. In order to prevent the typical pattern explosion in pattern mining, we employ closed patterns for focusing pattern exploration. In addition, we exploit efficient techniques for pruning the pattern space: We adapt a local variant of the standard Modularity metric used in community detection that is extended using optimistic estimates, and furthermore include graph abstractions. Our experiments on several standard datasets demonstrate the efficacy of our proposed novel method MinerLSD as an efficient method for local pattern mining on attributed networks.
\end{abstract}

Keywords: Complex networks, Attributed networks, Closed pattern mining, Network analysis and mining, Graph mining, Community detection

\section{Introduction}

The analysis of complex networks, e.g., by investigating structural properties and identifying interesting patterns, is an important task to make sense of such networks, in order to ultimately enable an understanding of their phenomena and structures, e.g., (Newman 2003; Kumar et al. 2006; Almendral et al. 2007; Mitzlaff et al. 2011; Silva et al. 2012; Mitzlaff et al. 2013; Atzmueller 2014; Pool et al. 2014; Galbrun et al. 2014; Mitzlaff et al. 2014; Kibanov et al. 2014; Soldano et al. 2015; Atzmueller et al. 2016; Bendimerad et al. 2016; Kaytoue et al. 2017; Atzmueller 2017; 2019). In this context, data mining on such networks represented as attributed graphs has recently emerged as a prominent research topic, e.g., (Moser et al. 2009; Silva et al. 2012; Atzmueller 2014; Galbrun et al. 2014; Soldano et al. 2015; Atzmueller et al. 2016; Bendimerad et al. 2016; Kaytoue et al. 2017). Methods for mining attributed graphs focus on the identification and extraction of patterns using topological information as well as compositional information on nodes and/or edges given by a set of attributes, e.g., (Atzmueller 2018; Wasserman and Faust 1994). In particular, local pattern mining focuses on the identification of dense substructures in a graph that are captured by specific patterns composed of the given attributes, e.g., for detecting communities (Moser et al. 2009; Silva et al. 2012; Pool et al. 2014; Galbrun et al. 2014; Soldano et al. 2015; Atzmueller et al. 2016).

(c) The Author(s). 2019 Open Access This article is distributed under the terms of the Creative Commons Attribution 4.0 International License (http://creativecommons.org/licenses/by/4.0/), which permits unrestricted use, distribution, and reproduction in any medium, provided you give appropriate credit to the original author(s) and the source, provide a link to the Creative Commons license, and indicate if changes were made. 
In this paper, an adapted and substantially extended revision of Atzmueller et al. (2018), we present MinerLSD a method for the efficient mining of local patterns on attributed networks. Compared to our work described in Atzmueller et al. (2018), we have added onto the discussion of the MinerLSD algorithm, also considering further related approaches for putting the proposed method into context. Furthermore, we have considerably extended the evaluation and discussion of the proposed novel algorithm with new experiments, also using new (larger) datasets, and by illustrating the pattern mining approach using exemplary patterns.

MinerLSD focuses both on local pattern mining (e.g., for local community detection) using the local modularity metric (Newman 2004; Newman and Girvan 2004; Atzmueller et al. 2016), as well as graph abstraction that reduces graphs to k-core subgraphs (Soldano et al. 2015). In order to prevent the typical pattern explosion in pattern mining, we employ closed patterns. In addition, we exploit optimistic estimates for the local modularity for focussing pattern exploration inspired by community detection methods and for pruning the pattern space. Essentially, the optimistic estimate technique provides two advantages: First, it neglects the importance of a minimal support threshold which is typically applied in pattern mining. Second, it enables a very efficient pattern exploration approach, given a suitable threshold for the local modularity, as we will show below. Then, this threshold can of course alternatively be entirely eliminated in a top-k approach. We demonstrate the efficacy of our presented novel method MinerLSD by performing experiments on several standard datasets, in relation to two baselines for local pattern mining.

Our contributions are summarized as follows:

1. For local pattern mining on attributed graphs, we analyze the impact of generating closed patterns compared to standard pattern mining in terms of the search effort.

2. Using two baseline algorithms, we further investigate the impact of pruning the pattern exploration space using an optimistic estimate of the local modularity measure with different thresholds.

3. Finally, we propose the MinerLSD method for efficient local pattern mining on attributed graphs. MinerLSD relies on closed pattern mining, optimistic estimate pruning, and graph abstraction.

The rest of this paper is organized as follows: Section "Related Work" discusses related work, before section "Background" introduces basic notions and concepts. After that, "The MinerLSD Algorithm" section presents the novel MinerLSD method. Next, section "Datasets" introduces the applied datasets. Section "Experiments and Results" discusses our experimental results. Finally, section "Conclusions" concludes with a summary and interesting directions for future work.

\section{Related Work}

The detection of local patterns is a prominent approach in knowledge discovery and data mining, e.g., (Morik 2002; Morik et al. 2005; Knobbe et al. 2008). Below, we discuss related work in the areas of local pattern mining, closed patterns, graph abstractions, and community detection on attributed graphs.

In particular, the proposed novel MinerLSD algorithm builds on methods for those fields. Thus, similar to the approaches discussed below, the proposed MinerLSD approach also utilizes closed patterns, and graph abstractions, i.e., core subgraphs. However, it 
extends this using optimistic estimate pruning using an interestingness measure adapted from (local) community detection. In section "Experiments and Results", we perform an extensive evaluation of the impact of closed patterns, optimistic estimates, and core structures on the pattern mining effort.

\section{Pattern Mining}

In general, local pattern mining, e.g., (Agrawal and Srikant 1994; Han et al. 2000; Morik 2002; Morik et al. 2005; Knobbe et al. 2008; Lemmerich et al. 2012; Atzmueller 2015; Lemmerich et al. 2016) has many flavors, including association rule mining, subgroup discovery, and graph mining. At its core, it considers the support set of any pattern, i.e., the set of objects, often called transactions, in which the pattern occurs. The goal then is to enumerate the set of all patterns that satisfy some constraint. In the case of association rules (Agrawal and Srikant 1994; Han et al. 2000) typically the frequency of a pattern, or the frequency of a contained implication in the pattern, respectively, are considered. Whenever the constraint is anti-monotonic, as the frequency, a top-down search may be efficiently pruned. Still this results in investigating a lot of patterns. In the field of subgroup discovery, more complex constraints formalizx ed in quality (or interestingness) functions have been proposed; here, these do not necessarily fulfill anti-monotonicity. To handle that, optimistic estimates for those quality functions have been proposed (Wrobel 1997; Grosskreutz et al. 2008; Atzmueller and Lemmerich 2009; Lemmerich et al. 2016) in order to efficiently prune the pattern search space. Closed pattern mining (see for instance (Pasquier et al. 1999)) reduces the search by considering patterns as equivalent when having the same support set, and generating only closed patterns, i.e., a most specific pattern among all equivalent patterns. Efficient enumeration algorithms have been provided, e.g., (Uno et al. 2004; Boley et al. 2010)). Various algorithms and methodologies using closure operators have also been proposed in the domain of formal concept analysis (Wille 1982), which goes further than the enumeration alone, being interested in the lattice structure of the set of closed patterns (Ganter and Wille 1999).

\section{Local Pattern Mining on Attributed Networks}

For investigating complex networks, a popular approach consists of extracting a core subgraph from the network, i.e., some essential part of the graph whose nodes satisfy a local property. The $k$-core definition was first proposed in Seidman (1983). It requires all nodes in the core subgraph to have a degree of at least $k$. The idea was further extended to a wide class of so-called generalized cores (Batagelj and Zaversnik 2011). The resulting subgraphs may be made of several connected components that are then considered as structural communities. However, as this may be too weak to obtain cohesive communities, some post-processing may then be necessary. A successful method, for example, identifies $k$-communities (Palla et al. 2005) that are extracted from the connected components of a graph derived from the original graph.

Recently an extension of the closed pattern mining methodology to attributed graphs has been proposed. It relies on the reduction of the support set of a pattern to the core of the pattern subgraph (Soldano and Santini 2014). This results in less and larger classes of equivalent patterns, and hence less closed patterns. The MinerLC algorithm proposed by Soldano et al. (2017) is a generic method to enumerate the set of such core closed patterns. The algorithm MinerLSD that we propose in "The MinerLSD Algorithm" section, closely 
follows the MinerLC algorithm and adds requirements regarding the local modularity of the pattern core subgraphs. This is performed efficiently using the optimistic estimate pruning strategy of the COMODO algorithm for community detection, mentioned in section "Community Detection on Attributed Graphs".

\section{Community Detection on Attributed Graphs}

Communities and cohesive subgroups have been extensively studied in network science, e.g., using social network analysis methods (Wasserman and Faust 1994). Fortunato (2010) presents a thorough survey on the state of the art community detection algorithms in graphs, focussing on detecting disjoint communities, e.g., (Newman and Girvan 2004; Fortunato and Castellano 2007). In contrast to such partitioning approaches, overlapping communities allow an extended modeling of actor-actor relations in social networks: Nodes of a corresponding graph can then participate in multiple communities, e.g., (Palla et al. 2007; Lancichinetti et al. 2009; Xie and Szymanski 2013). A comprehensive survey on algorithms for overlapping community detection is provided in Xie et al. (2013). In contrast to the algorithms and approaches discussed above, the proposed approach utilizes further descriptive information of attributed graphs, e.g., (Bothorel et al. 2015).

Attributed (or labeled) graphs as richer graph representations enable approaches that specifically exploit the descriptive information of the labels assigned to nodes and/or edges of the graph. Exemplary approaches include density-based methods, e.g., (Zhou et al. 2009; Combe et al. 2015), distance-based methods, e.g., (Steinhaeuser and Chawla 2008; Ge et al. 2008), entropy-based methods, e.g., (Zhu et al. 2011; Smith et al. 2014), model-based methods, e.g., (Balasubramanyan and Cohen 2011; Xu et al. 2012), seedcentric methods, e.g., (Kanawati 2014a; Yakoubi and Kanawati 2014; Kanawati 2014b; Belfin et al. 2018) and finally pattern mining approaches, which we will describe in the following in more detail.

Pattern mining approaches for community detection on attributed graphs typically connect (local) pattern mining and community detection according to several interestingness measures or optimization criteria. Moser et al. (2009), for example, combine the concepts of dense subgraphs and subspace clusters for mining cohesive patterns. Starting with quasi-cliques, those are expanded until constraints regarding the description or the graph structure are violated. Similarly, Günnemann et al. (2013) combine subspace clustering and dense subgraph mining, also interleaving quasi-clique and subspace construction. Galbrun et al. (2014) propose an approach for the problem of finding overlapping communities in graphs and social networks, that aims to detect the top-k communities so that the total edge density over all $\mathrm{k}$ communities is maximized. This is also related to a maximum coverage problem for the whole graph. For labeled graphs, each community is required to be described by a set of labels. The algorithmic variants proposed by Galbrun et al. apply a greedy strategy for detecting dense subgroups, and restrict the resulting set of communities, such that each edge can belong to at most one community. This partitioning involves a global approach on the community quality, in contrast to our local approach. Silva et al. (2012) study the correlation between attribute sets and the occurrence of dense subgraphs in large attributed graphs. The proposed method considers frequent attribute sets using an adapted frequent item mining technique, and identifies the top-k dense subgraphs induced by a particular attribute set, called structural correlation patterns. The DCM method presented by Pool et al. (2014) includes a two-step process of community 
detection and community description. A heuristic approach is applied for discovering the top-k communities, utilizing a special interestingness function which is based on counting outgoing edges of a community similar; for that, they also demonstrate the trend of a correlation with the Modularity function.

The COMODO algorithm proposed by Atzmueller et al. (2016) applies an adapted subgroup discovery (Atzmueller and Puppe 2006; Atzmueller 2015) approach for community detection on attributed graphs. That is, COMODO applies subgroup discovery for detecting interesting patterns (constructed from the set of compositional attributes) for which their interestingness is evaluated on the graph topological structure. The algorithm works on an edge dataset that is attributed with common attributes of the respective nodes. Then, communities are detected in a top- $\mathrm{k}$ approach maximizing a given community interestingness measure. This includes, among others, the local modularity, which is derived from the (global) measure, i.e., the (Newman) Modularity (Newman 2004; Newman and Girvan 2004). For an efficient community detection approach, COMODO utilizes optimistic estimate pruning.

In this paper, we adapt the COMODO approach integrating optimistic estimate pruning for the local modularity as proposed by COMODO with closed abstract pattern mining of the MinerLC algorithm. This results in the efficient and effective MinerLSD algorithm, making use of efficient techniques based on abstract closed pattern mining and branch-and-bound pruning according to the local modularity. At the same time, these techniques allow effective selection strategies utilizing graph abstractions together with local modularity, as we will show below.

\section{Background}

In the following, we outline the background on closed local pattern mining, introduce pruning based on optimistic estimates, and discuss pattern exploration, abstraction, and selection combining principles from pattern mining and graph mining, i.e., utilizing closure on the attribute space and topological criteria based on local modularity (estimates) and $\mathrm{k}$-cores.

\section{Mining Closed Patterns to Enumerate Core Subgraphs}

We consider the following general problem: Let $G$ be an attributed graph, i.e., a graph where each vertex $v$ is described by an itemset $D(v)$ taken from a set of items $I$. We want to enumerate all (maximal) vertex subsets $W$ in $G$ such that there exists an itemset $q$ which is a subset of all itemsets $D(v), v \in W . W$ is furthermore required to satisfy some graph related constraints. In standard terminology, $q$ is a pattern that occurs in all element of $W$ which is also called the support set or extension ext(q) of $q$. Efficient top-down enumeration algorithms exist as far as the constraints are anti-monotonic: whenever the constraint fails to be satisfied by some pattern, it also fails for all more specific patterns. This is obviously the case for the minimum support constraint that requires the size of $\operatorname{ext}(q)$ to be above some minimal support threshold $s$.

A first way to reduce the overall search space and the size of the solution set is to avoid duplicates, i.e., patterns $q, q^{\prime}$ that occur in the same subgroup, for which $\operatorname{ext}(q)=\operatorname{ext}\left(q^{\prime}\right)$. This is obtained by only enumerating closed patterns. Given any pattern $q$ the associated closed pattern is the most specific pattern $f(q)$ which occurs in the same subgroup as $q$, i.e., $\operatorname{ext}(f(q))=\operatorname{ext}(q)$. Furthermore, since we consider the vertices of a graph, it is natural 
to consider graph related constraints, as for instance requiring that all vertices have a degree of at least $k$ in the subgroup graph $G_{W}$. For that purpose, each candidate subgroup $X$ is reduced to its core $p(X)=W$ using the core operator $p$.

We start with the definition of closure: The operator $f$ that returns for any pattern $q$ the closed pattern $f(q)$ is a closure operator (see below) defined by $f(q)=\operatorname{int} \circ p \circ \operatorname{ext}(q)$; the respective operators are defined as follows (note that o denotes function composition):

- The intersection operator $\operatorname{int}(X)$ returns the most specific pattern occurring in the vertex subset $X$.

- The core operator $p(X)$ returns the core, according to some core definition, of the subgraph $G_{X}$ of $G$ induced by the vertex subset $X . p$ is an interior operator (see below).

Definition 1 Let $S$ be an ordered set and $f: S \rightarrow S$ a self map such that for any $x, y \in S$, $f$ is monotone, i.e. $x \leq y$ implies $f(x) \leq f(y)$ and idempotent, i.e. $f(f(x))=f(x)$ :

- If $f(x) \geq x, f$ is called a closure operator.

- If $f(x) \leq x, f$ is called an interior operator.

Essentially, core closed pattern mining relies on three main results:

1. It has been shown that whenever $p$ is an interior operator, $f=\operatorname{int} \circ p \circ$ ext is a closure operator (Pernelle et al. 2002).

2. Furthermore, core definitions rely on a monotone property of a vertex within an induced subgraph (Batagelj and Zaversnik 2002). For instance, the $k$-core of a subgraph $G_{X}$ is defined as the largest vertex subset $W \subseteq X$ such that in the induced subgraph $G_{W}$ all vertices $v$ have a degree of at least $k$. The property is monotone in the sense that when increasing $G_{X}$ to $G_{X^{\prime}}$ the degree of $v$ cannot decrease.

3. Finally, it has been shown that the core operator which returns the core of some subgraph $G_{X}$, according to a monotone property, is an interior operator (Soldano and Santini 2014).

Overall, this means that $f(q)$ returns the largest pattern which occurs in the core of the vertex subset $\operatorname{ext}(q)$ in which $q$ occurs. This is exploited in core closed pattern mining (Soldano et al. 2017), performing a top-down search of the pattern space jumping from closed pattern to closed pattern: each closed pattern $q$ is augmented with some item $x$, then the next closed pattern $f(q \cup\{x\})$ is computed.

\section{Pruning Local Patterns in Graphs Using Optimistic Estimates}

Another way to reduce the solution set is to consider some interestingness measure $M$ and require a subgroup $W$ to induce a subgraph $G_{W}$ with an interestingness $M(W)$ above some threshold. However such measures, for example, the local modularity (see below), are usually not anti-monotonic. This difficulty may be overcome by using some optimistic estimate of $M$ which is both anti-monotonic and allows an efficient pruning of the search space. Optimistic estimates are one prominent option in local pattern mining to prune search spaces by complementing non-(anti)-monotonic interestingness measures by their respective optimistic estimators, e.g., (Grosskreutz et al. 2008; Wrobel 1997). Intuitively, if for a given pattern (and all of its potential specializations) it can be proven that their quality is either below the quality of the current top patterns, or below a specified threshold, 
then pattern exploration does not need to continue for that pattern, and the search space can often be pruned significantly.

In the scope of local pattern mining on graphs, several standard community quality functions have been investigated, also specifying optimistic estimates for a number of such community evaluation functions. As shown in Atzmueller et al. (2016) these lead to a quite efficient approach for descriptive community detection using local pattern mining. In summary, using optimistic estimates we can enumerate pairs $(c, W)$, of pattern $c$ and subgroup $W$ inducing the subgraph $G_{W}$. Then, we can select subgraphs according to an interestingness measure $M$ of the subgraph using an anti-monotonic optimistic estimate of $M$ to prune the search. Additionally, a minimal support constraint can also be applied in order to improve the effectiveness of pruning.

Below, we summarize main results on using optimistic estimate pruning for community detection, specifically addressing the (local) modularity quality measure. Here, the concept of a community intuitively describes a group $W$ of individuals out of a population such that members of $W$ are strongly "connected" to each other but sparsely "connected" to those individuals that are not contained in $W$. This notion translates to communities as vertex sets $W \subseteq V$ of an undirected graph $G=(V, E)$; in the following, we adopt the notation of Atzmueller et al. (2016) for introducing the main concepts: $n:=|V|, m:=|E|$, and $m_{W}:=|\{\{u, v\} \in E: u, v \in W\}|$ denotes the number of intra-edges of $W$.

There are different interestingness measures for estimating the quality of a community $2^{V} \rightarrow \mathbb{R}$, also according to different criteria and intuitions about what "makes up" a good community. One particular community quality function is the Modularity (Newman 2004; Newman and Girvan 2004). In the context of local pattern mining, we aim to maximize local quality functions for single communities. For that, we apply an adaptation of the Modularity interestingness measure, which essentially is a global measure estimating the quality of a community partitioning. Then, we focus on the modularity contribution of each individual community in order to obtain a local measure for each community, cf., (Atzmueller et al. 2016), which we further call local modularity (MODL).

Overall, the Modularity MOD (Newman 2004; Newman and Girvan 2004; Newman 2006) of a graph clustering with $k$ communities $C_{1}, \ldots, C_{k} \subseteq V$ focuses on the number of edges within a community and compares that with the expected such number given a nullmodel (i.e., a corresponding random graph where the node degrees of $G$ are preserved). It is given by

$$
\mathrm{MOD}=\frac{1}{2 m} \sum_{u, v \in V}\left(A_{u, v}-\frac{\mathrm{d}(u) \mathrm{d}(v)}{2 m}\right) \delta(C(u), C(v)),
$$

where $C(i)$ denotes for $i \in V$ the community to which node $i$ belongs. $A_{u, v}$ denotes the respective entry of the adjacency matrix $A . \delta(C(u), C(v))$ is the Kronecker delta symbol that equals 1 if $C(u)=C(v)$, and 0 otherwise.

The modularity contribution of a single community given by a vertex set $W, W \subseteq V$ in a local context (e.g., in a subgraph induced by the pattern), i.e., the local modularity (MODL), can then be computed (cf., (Newman 2006; Nicosia et al. 2009; Atzmueller et al. 2016)) as follows:

$$
\operatorname{MODL}(W)=\frac{m_{W}}{m}-\sum_{u, v \in W} \frac{\mathrm{d}(u) \mathrm{d}(v)}{4 m^{2}} .
$$


For the above (MODL), an optimistic estimate has been introduced in Atzmueller et al. (2016). It can be derived based only on the number of edges $m_{W}$ within the community:

$$
\operatorname{oe}(\operatorname{MODL})(W)= \begin{cases}0.25, & \text { if } m_{W} \geq \frac{m}{2}, \\ \frac{m_{W}}{m}-\frac{m_{W}^{2}}{m^{2}}, & \text { otherwise. }\end{cases}
$$

For a detailed discussion, the derivation of the local measure, and the respective proofs, we refer to Atzmueller et al. (2016).

\section{Local Pattern Exploration, Abstraction, and Selection}

Pattern mining commonly aims at discovering a set of novel, potentially useful, and ultimately interesting patterns from a given (large) data set (Fayyad et al. 1996). For pattern exploration, we apply local pattern mining, in particular, (abstract) closed pattern mining (Pasquier et al. 1999; Uno et al. 2004; Boley et al. 2010; Soldano and Santini 2014; Soldano et al. 2017) due to its efficient traversal of the search space for pattern enumeration and abstraction as discussed above.

Regarding pattern selection, we discuss the choices of core abstraction and modularitybased selection in the following: In contrast to many methods used in network analysis and graph mining, pattern mining on attributed graphs specifically aims at a descriptionoriented view, by including patterns on attributes, but also considering the topological structure. Many community mining algorithms, for example, only collect sets of nodes denoting the individual communities thus merely focusing on structural/topological aspects of the graph; typically, then there is no simple and easily interpretable description, such that a community would be represented mainly as a set of IDs, cf., (Atzmueller et al. 2016).

For local pattern mining, the goal is typically to detect a set of the most interesting patterns according to a given quality function, e.g., with a quality above a certain threshold, or the top-k patterns according to the ranking of the quality function denoting their interestingness. For subgroup discovery, as an exemplary instance, the goal is then to obtain the set of patterns covering subgroups that are "as large as possible and have the most unusual statistical characteristic with respect to the property of interest" (Wrobel 1997). Thus, the interestingness of a pattern can then be flexibly defined, e.g., by a significant deviation from a model that is derived from the total population (Morik 2002; Morik et al. 2005; Knobbe et al. 2008). Therefore, typically the size of a pattern or the size of its extension, respectively, and the deviation compared to some null-model specifies the interestingness which is formalized in the quality function for ranking the patterns.

For pattern mining on networks and graphs, there exist several quality measures, usually taking into account the support of the pattern, i.e., its size, similar to the criteria discussed above. Furthermore, the topological structure of the subgraph induced by the pattern is also taken into account. Here, standard quality functions include the segregation index (Freeman 1978), the average out degree fraction (Yang and Leskovec 2012), the conductance (Leskovec et al. 2008) and the Modularity (Newman and Girvan 2004), as we have discussed in the previous section. In general, the core idea of the evaluation function is to apply an objective evaluation criterion, for example, for the Modularity the number of connections within the community compared to the statistically "expected" number based on all available connections in the network, and to prefer those communities that optimize the evaluation function. 
A thorough empirical analysis of the impact of different community mining algorithms and their corresponding objective function on the resulting community structures is presented in Leskovec et al. (2010), based on the analysis of community structure in graphs (as presented in Leskovec et al. (2008)). Furthermore, Atzmueller et al. (Atzmueller and Mitzlaff 2010; 2011; Atzmueller et al. 2016) have empirically investigated different community quality functions in the scope of local pattern mining. As shown there for the provided experiments, the local modularity quality function indicated the best results for pattern filtering and pruning in local pattern mining applications, since it provides large high quality communities, i.e., subgroups referring to the induced subgraphs, smaller patterns in terms of their description, as well as statistically significant patterns compared to the other mentioned quality functions which focus on smaller subgroups; those were typically also not statistically significant as specifically presented in Atzmueller et al. (2016).

Furthermore, the local modularity quality function (see Eq. 2) intuitively provides the prominent property of assigning a higher ranking to larger (core) subgraphs under consideration, if these are considerably more densely connected than expected by chance. Therefore, these criteria conveniently capture the notion of larger subgraphs and having the most unusual statistical characteristics with respect to the null-model. In the following, we show how these criteria are directly implemented in the local modularity measure.

Consider the local modularity $\operatorname{MODL}(W)$ of a subgraph $W$ :

$$
\operatorname{MODL}(W)=\frac{m_{W}}{m}-\sum_{u, v \in W} \frac{\mathrm{d}(u) \mathrm{d}(v)}{4 m^{2}}=\frac{1}{m}\left(m_{W}-\sum_{u, v \in W} \frac{\mathrm{d}(u) \mathrm{d}(v)}{4 m}\right) .
$$

Since the first factor $\frac{1}{m}$ is a constant, we can consider the second factor of the former expression: It is easy to see that this factor itself is order equivalent to the local modularity function MODL, since it only depends on a fixed constant $\frac{1}{\mathrm{~m}}$; by not including that it is thus not normalized relatively to the number of edges of the graph. Instead, it focuses on the number of edges of the (core) subgraph (the minuend of the term) and its deviation assessed by the null-model which is captured by the subtrahend of that term.

Thus, it is easy to see that the MODL function tends to focus on larger patterns (larger subgraphs) having the most unusual statistical characteristics with respect to the nullmodel. By utilizing appropriate constraints on the graph structure, e.g., using k-core abstractions we can further focus on the unusual distributional characteristics. By applying $k$-core abstractions, for example, with increasing $k$ we tend to focus on increasingly denser pattern structures (subgraphs). We will also show this by our experiments in section "Experiments and Results" when we discuss our results.

To sum up, we apply the local modularity measure MODL as introduced above for focusing pattern exploration on the statistically most unusual subgraphs. Applying $k$-core constraints helps due to its focus on denser subgraphs, as also theoretically analyzed in Peng et al. (2014) for $k$-cores. Overall, we specifically focus on "nuggets in the data" (Klösgen 1996), i.e., on exceptional patterns according to the principles of local pattern mining. In addition, the local modularity neglects the importance of a minimal support threshold 
which is typically applied in pattern mining, since it directly includes the size of the pattern as a criterion. This enables a very efficient pattern mining approach, given either a suitable threshold for the local modularity, or by targeting the top- $k$ patterns.

\section{The MinerLSD Algorithm}

In the following, we describe our proposed novel method MinerLSD in detail. MinerLSD integrates core subgraph closed pattern mining with pattern selection according to the local modularity MODL function, and optimistic estimate pruning according to a specific optimistic estimator, i.e., oe(MODL).

As input parameters, MinerLSD requires a graph $G=(V, E)$, a set of items $I$, a dataset $D$ describing vertices as itemsets and a core operator $p \cdot p$ depends on $G$ and to any image $p(X)=W$ we associate the core subgraph $C$ whose vertex set is vs $(C)=W$. In our experiments, $p(X)$ returns the k-core of $X$. As further parameters, MinerLSD considers the corresponding value $k$ as well as a frequency threshold $s$ (defaulting to 0 ) and a local modularity threshold $l m$. The algorithm outputs the frequent pairs $(c, W)$ where $c$ is a core closed pattern and $W=p \circ \operatorname{ext}(c)$ its associated k-core. For evaluation purposes, we also count the number of patterns above the local modularity threshold (\#1m), and the number of patterns for which their estimate is above the local modularity threshold (\#lme). It is important to note, that in the enumeration step MinerLSD ensures that each pair $(c, W)$ is enumerated (at most) once.

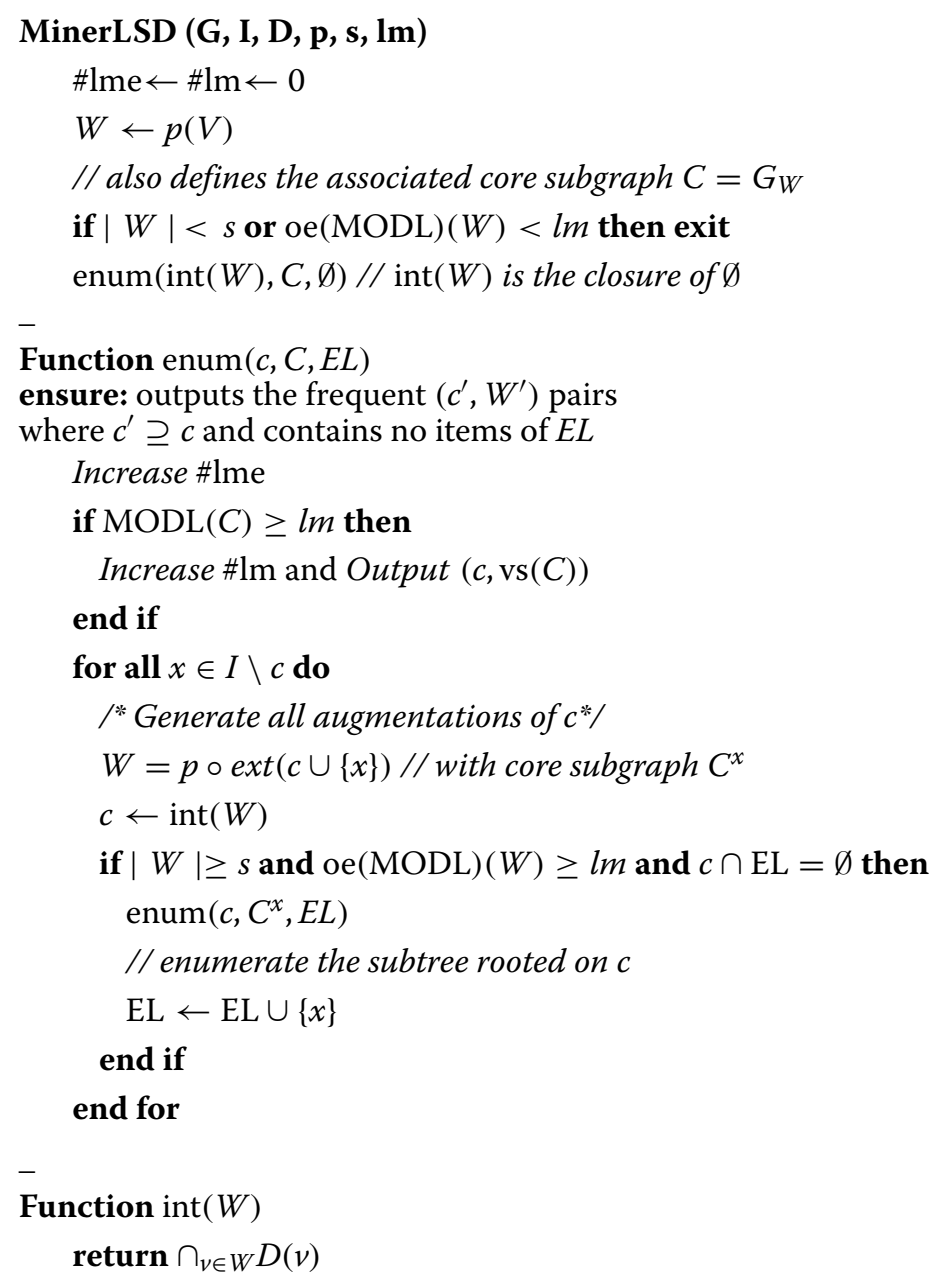




\section{Datasets}

We performed our experiments utilizing a variety of attributed graph datasets ranging from small to medium graphs with small to large sets of items. Table 1 depicts the main characteristics of these datasets (see also (Galbrun et al. 2014)), which have been previously used in pattern mining tasks on attributed graphs. For each dataset, we indicate the number of edges $(|E|)$, vertices $(|V|)$ and labels $(|L|)$, the average vertex degree $(\overline{\operatorname{deg}(v)})$ and average number of labels per vertex $(\overline{|l(v)|})$ in the table.

- S50 is a standard attributed graph dataset used in a previous work about graph abstractions (Soldano and Santini 2014). ${ }^{1}$ It represents 148 friendship relations between 50 pupils of a school in the West of Scotland; the labels concern the students' substance use (tobacco, cannabis and alcohol) and sporting activity. The values of the corresponding variables are ordered (see (Soldano and Santini 2014) for details).

- The Lawyers dataset concerns a network study of corporate law partnership that was carried out in a Northeastern US corporate law firm from 1988 to 1991 in New England (Lazega 2001). It concerns 71 attorneys (partners and associates) of this firm who are the vertices of four networks. In the resulting data, each attorney is described using various attributes. ${ }^{2} \mathrm{We}$ consider the advice network which is originally a directed graph in a undirected version, so that two lawyers are connected if at least one asks for advice to the other one.

- The CoExp dataset models a representative regulatory network for yeast obtained from Microarray expression data processed by the CoRegNet(Nicolle et al. 2015) program. In the CoExp dataset the vertices are co-regulators and they are linked if they share a common set of target genes. The vertices are labeled with their influence profile along a metabolic transition of the organism. Each influence value represents the regulation activity of the considered co-regulator at some instant of the metabolic transition.

- LastFM, DBLP.C and DBLP.XL were used in Galbrun et al. (2014). LastFM models the social network of last.fm where individuals are described by the artists or groups they have listened to. DBLP.C contains a co-authorship graph built from a set of publication references extract from DBLP of researchers that have published in the ICDM conference. The authors are labeled by keywords extracted from the papers' titles. DBLP.XL is the complete labeled DBLP co-authorship network used in Galbrun et al. (2014).

Table 1 Datasets/Characteristics: Number of edges (|E|), vertices (|V|), labels (|L|), the average vertex degree $(\overline{\operatorname{deg}(v)})$, and average number of labels per vertex $(\overline{|/(v)|})$

\begin{tabular}{llllll}
\hline Nom & $|\boldsymbol{V}|$ & $|\boldsymbol{E}|$ & $|\boldsymbol{L}|$ & $\overline{\boldsymbol{d e g}(\boldsymbol{v})}$ & $\overline{\boldsymbol{l}(\boldsymbol{v}) \boldsymbol{|}}$ \\
\hline S50 & 50 & 74 & 14 & 2.96 & 7 \\
Lawyers & 71 & 556 & 42 & 15.66 & 20 \\
CoExp & 151 & 1849 & 36 & 24.49 & 18 \\
LastFM & 1892 & 12717 & 17625 & 13.44 & 40.07 \\
Delicious & 1867 & 7664 & 52800 & 8.21 & 123.47 \\
DBLP.C & 3140 & 10689 & 4588 & 6.81 & 15.02 \\
DBLP.P & 45131 & 228173 & 32 & 10.11 & 2.15 \\
DBLP.S & 108032 & 276658 & 23254 & 5.12 & 13.93 \\
DBLP.XL & 929937 & 3461697 & 92164 & 7.44 & 10.16 \\
\hline
\end{tabular}


- DBLP.P was used in Bechara-Prado et al. (Bechara Prado et al. 2013). It represents a co-authorship graph built from a set of publication references extract from DBLP, published between January 1990 and February 2011 in the major conferences or journals of the Data Mining and Database communities. Three labels have been added to the original dataset based on the scope of the conferences and journals, respectively: DB (databases), DM (data mining) and AI (artificial intelligence).

- Delicious consists of the social (friendship) network of the resource sharing system delicious where individuals are described by their bookmarks' tags. The dataset is publicly available and was obtained from the HetRec workshop (Cantador et al. 2011) at Recsys $2011 .^{3}$

- DBLP.S was used in Silva et al. (2012). It also represents a co-authorship network from a set of publication references extracted from DBLP.

\section{Experiments and Results}

In the following, we first summarize the applied baseline methods that were used in the comparison with the presented MinerLSD method. After that, we present our experimental results on the datasets described in "Datasets" section.

\section{Baseline Methods}

The applied set of baseline methods consists of MinerLC - an efficient algorithm for mining core closed patterns, and COMODO - an efficient algorithm for descriptive community detection using optimistic estimates.

\section{MinerLC}

MinerLC ${ }^{4}$ (cf., (Soldano et al. 2017)) enumerates pairs (c,W) where $G_{W}$ is the core subgraph of pattern $c$, i.e., subgroup $W=p \circ \operatorname{ext}(c)$ where $\circ$ is the composition operator, $p$ is a core operator and $c$ is the largest pattern that occurs in $W$ and is called a core closed pattern. A threshold on the core sizes allows to select frequent core closed patterns and to accordingly prune the search. The selection process relies then partly on the anti-monotonic support constraint and partly on the fact that there are less pattern core subgraphs than pattern subgraphs as various pattern subgraphs $G_{\text {ext(q) }}$ may be reduced to the same core subgraph.

\section{COMODO}

The COMODO algorithm ${ }^{5}$ presented in Atzmueller et al. (2016) performs descriptionoriented community detection in order to discover the top- $k$ communities. In summary, COMODO enumerates pairs $(c, W)$ where $G_{W}$ is the subgraph of pattern $c$ for vertex subset $W$. It selects top $k$ subgraphs according to an interestingness measure $M$ of the subgraph and uses an efficient anti-monotonic optimistic estimate of $M$ to prune the search. Additionally, a minimal support constraint can also be applied in order to improve the effectiveness of pruning.

\section{Similarities and Differences in Pattern Selection}

Both the considered baseline methods, i.e., MinerLC and COMODO output a set of pairs (pattern, vertex subset). However, in order to compare their outputs we have to consider the following differences:

- In COMODO the vertex subset $W$ is obtained as the extremities of the set of edges in which a pattern occurs and a pattern occurs in an edge whenever it occurs, in the 
original dataset, in both connected vertices. That is, for each edge we assign the set of common items of both nodes, such that a pattern always covers two nodes connected by an edge. As a consequence, $W$ ignores isolated nodes in which $p$ occurs. To obtain the same vertex subset in MinerLC (and MinerLSD) it is necessary to remove isolated nodes, which is enabled by applying a 1-core graph abstraction.

- Since COMODO does not enumerate closed patterns, the same subgroup may be associated to several patterns. For that case, a post-processing is needed to eliminate the duplicates from the list of subgroups which may then be compared to the subgroups in the MinerLC pairs. This post-processing is one of the standard post-processing options of COMODO.

- MinerLC is run with a core definition while COMODO uses various parameters to limit the enumeration, as for instance the top-k parameter.

To compare the results, MinerLC (as well as MinerLSD) should be run with the same minimum support threshold as COMODO and should only use a 1-core abstraction. The other parameters of COMODO should then have a value that does not limit the enumeration, e.g., by providing a sufficiently large top- $k$ parameter to enable an exhaustive enumeration.

Furthermore, MinerLC and COMODO select patterns according to different criteria. This is exemplified in Fig. 1, in which we have three graphs and three subgraphs induced by three vertices (in red). The subgraph $G_{123}$ of the top graph $G$ is a 2-core with a local modularity of 0.178 . Within the central graph, the subgraph $G_{123}$ is also a 2-core but with a low local modularity of -0.15 . Finally, within the bottom graph, $G_{123}$ is not a 2-core (since it has an empty 2-core subgraph) with a high local modularity of 0.16 .

\section{Results and Discussion}

In our experiments below, we first investigate the impact of closure, before we focus on the k-core abstraction. We perform a detailed analysis of the efficiency of using the local modularity estimate for pruning the search space. Finally, we provide a structural pattern set analysis considering different metrics, and discuss exemplary patterns for illustrating the efficacy of the proposed approach.

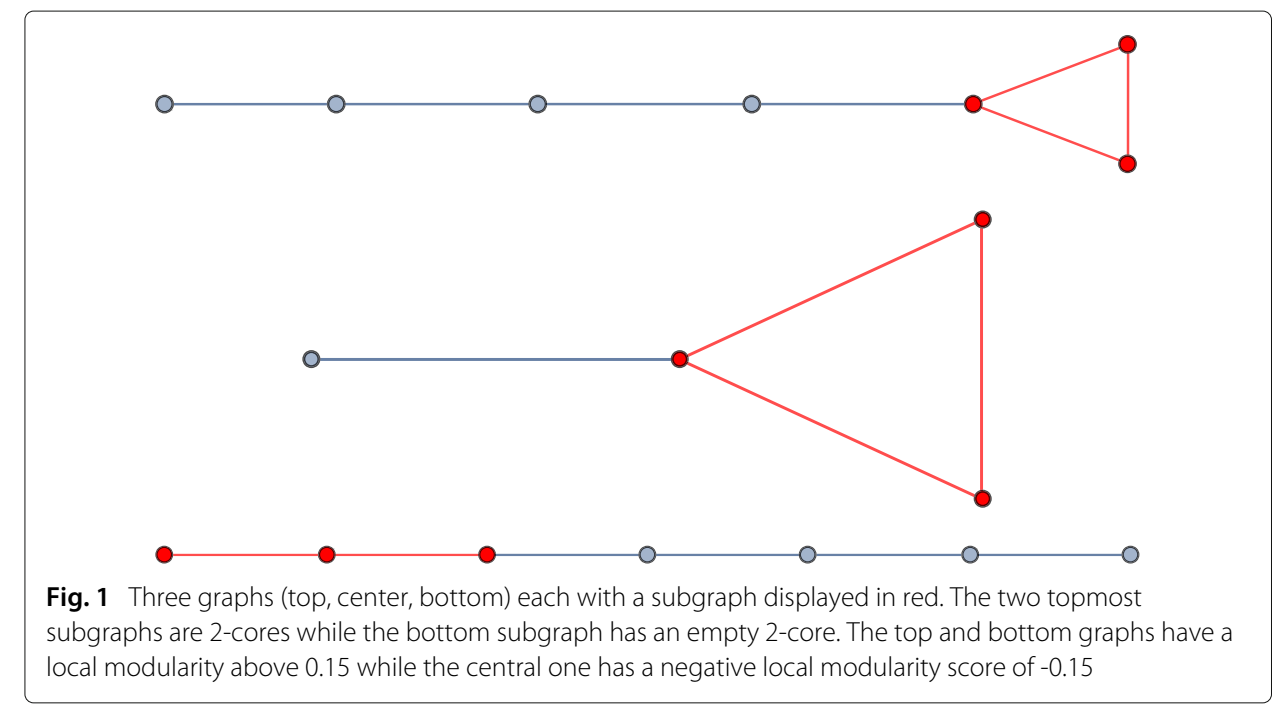




\section{Parameters and Datasets}

For MinerLSD, it is important to note that in our experiments described below we did not have to use the minimal support $s$, since the local modularity threshold is efficient enough to strongly reduce the number of patterns.

Below, we consider the following pattern quantities, where the (closed pattern, support set) pairs $(c, e)$ are output by MinerLC unless specified; also, we consider a given local modularity threshold $l m$.

- \#c the number of pairs $(c, e)$.

- \#lme: the number of pairs $(c, e)$ such that oe(MODL) $(e) \geq l m$.

- \#nec: the number of (necessary) pairs $(c, e)$ a top-down search has to consider to ensure that no pair with oe(MODL) $(e) \geq \operatorname{lm}$ is lost. See "Pruning: Efficiency of the Local Modularity Estimate" section for details and results on \#nec.

- \#lm the number of pairs $(c, e)$ such that $\operatorname{MODL}(e) \geq l m$

- \#lmeSD: the number of pairs $(c, e)$ such that oe(MODL) $(e) \geq l m$ as generated by COMODO.

We ran the original COMODO and MinerLC programs as available. MinerLSD is derived from the sources of MinerLC and is to be found on the MinerLC web site ${ }^{6}$. A new MinerLC version integrates the MinerLSD developments. The experimental results presented here may then be obtained using appropriate parameters and options of the new software.

\section{Impact of Closed Patterns in Reducing the Search Space}

MinerLSD searches a space of closed patterns while COMODO searches the whole pattern space. Therefore, we will investigate the impact of the closure reduction, for each local modularity threshold $l m$. For that, we first consider the quantity \#lme of core closed patterns with a local modularity estimate above $l m$, as provided by MinerLSD, when using 1-cores. We consider then the quantity \#lmeSD of patterns developed by COMODO using the same threshold. Table 2 reports \#lme and \#lmeSD for our datasets under investigation.

We observe two very different situations. In the Lawyers and CoExp datasets there is a large difference between \#lmeSD and \#lme, while there are considerable but not so strongly expressed differences in the other datasets compared to the former. Large differences typically occur when items have strong dependencies hence leading to a large reduction of the search space when applying a closure operator. For instance, in the Lawyers dataset vertices are described by various numeric attributes. In our representation, a single numeric attribute $x$ leads to a set of $x \leq s_{i}$ and of $x>s_{i}$ items with various thresholds $s_{i}$. This allows to include interval constraint as $\left.\left.x \in\right] s_{j}, s_{k}\right]$ within patterns. However there are then several equivalent patterns in which the same interval is represented in various ways. For instance, consider 4 thresholds $s_{1}, \ldots, s_{4}$, the interval $\left.\left.x \in\right] s_{2} s_{3}\right]$ is represented by $x>s_{2}, x \leq s_{3}, x>s_{1}, x>s_{2}, x \leq s_{3}$ and $x>s_{1}, x>s_{2}, x \leq s_{3}, x \leq s_{4}$. The latter is the only one found in a closed pattern. COMODO has then to generate many equivalent patterns while MinerLC, which applies a closure operator at each specialization step never generates two equivalent patterns, thus reducing the exploration of the pattern space effectively. 
Table 2 Number of patterns to develop in MinerLSD and COMODO (according to the respective local modularity threshold 0.005 ... 0.15) using a 1-core abstraction for MinerLSD

\begin{tabular}{|c|c|c|c|c|c|c|}
\hline Data / \#c & 0.005 & 0.01 & 0.02 & 0.03 & 0.05 & 0.15 \\
\hline $\mathrm{S} 50$ & 83 & & & & & \\
\hline \#ImeSD & 493 & 493 & 357 & 326 & 259 & 83 \\
\hline \#Ime & 83 & 83 & 77 & 72 & 67 & 36 \\
\hline CoExp & 196 & & & & & \\
\hline \#ImeSD & 1232895 & 991231 & 806911 & 468991 & 285183 & 77823 \\
\hline \#Ime & 178 & 166 & 150 & 133 & 114 & 64 \\
\hline DBLP.P & 2396 & & & & & \\
\hline \#ImeSD & 148 & 32 & 18 & 9 & 5 & 3 \\
\hline \#Ime & 34 & 22 & 15 & 9 & 5 & 3 \\
\hline Lawyers & 3221 & & & & & \\
\hline \#ImeSD & 3021675 & 1535949 & 677089 & 420699 & 168689 & 10339 \\
\hline \#Ime & 2929 & 2512 & 1970 & 1640 & 1146 & 295 \\
\hline DBLP.C & 14820 & & & & & \\
\hline \#ImeSD & 179 & 66 & 24 & 16 & 7 & 1 \\
\hline \#lme & 179 & 66 & 24 & 16 & 7 & 1 \\
\hline
\end{tabular}

In the DBLP.P datasets at the contrary the items are tags, with no taxonomic order relating them. Therefore, the values of \#lme and \#lmeSD are much closer, and even identical regarding the DBLP.C dataset.

\section{$k$-core sizes of the various networks}

Before considering how reducing support sets to k-cores affects the number of closed patterns in each dataset, we consider the various networks and compute their k-core sizes for a range of values of $k$. This pre-analysis aims to evaluate which level of $k$ we should use in our experiments. For small datasets for which computing closed patterns does need much resources this is not that important. However, for large datasets with many attributes, i.e., potentially large numbers of closed patterns, it is much better to have a rough guideline for selecting appropriate parameters for optimizing the computational effort.

In Fig. 2 we display the k-core sizes for a range of values of $k$, for each dataset. As we will see below, the small but densest networks for which local-modularity-based pruning has a weak efficiency, namely coExp and Lawyers, also exhibit a (relatively) slow decay with respect to increasing $k$ values, whereas for the other (larger) datasets we observe a quite considerable decrease in terms of the k-core sizes.

\section{Modularity Distributions}

As a prerequisite for the further analysis of the local modularity optimistic estimate, we aimed to get a more detailed insight into the distribution, similar to our pre-analysis for the k-cores discussed above. Figures 3-4 show the detailed results. The plots indicate the "meaningful" values for estimating the local modularity thresholds, which support our selections of parameters in the subsequent evaluations. Furthermore, Fig. 3 also indicates the pruning potential of the local modularity threshold, even using our rather approximating sampling strategy.

\section{Pruning: Efficiency of the Local Modularity Estimate}

For investigating the efficiency of pruning using the modularity estimate, we compare our proposed algorithm MinerLSD to the MinerLC algorithm, which applies no optimistic 


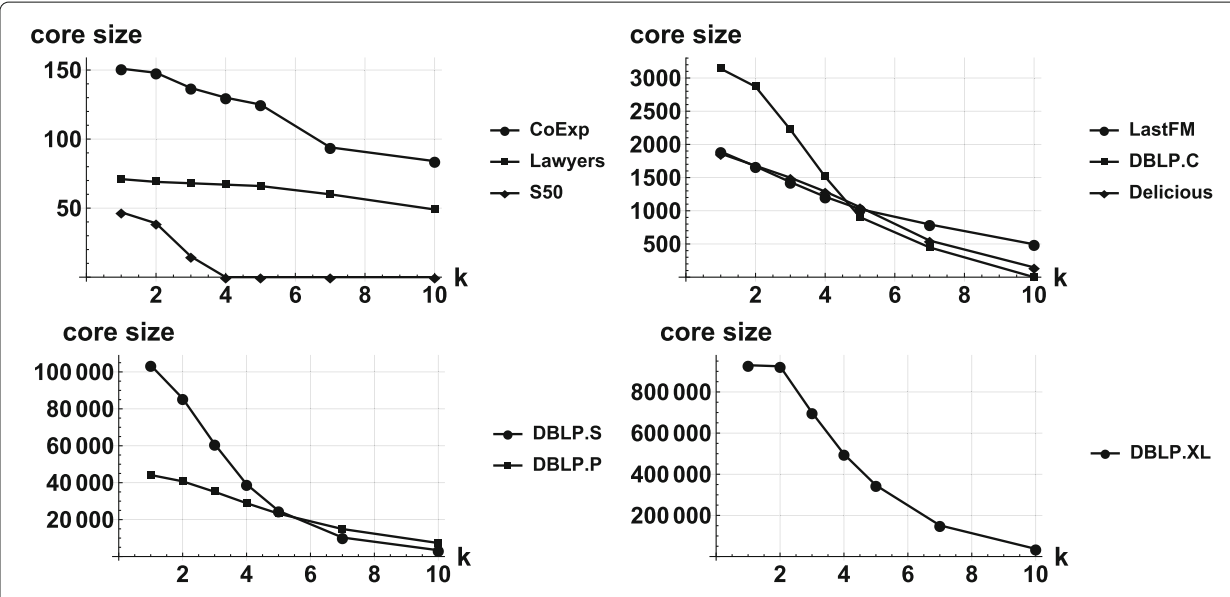

Fig. 2 k-core sizes of the networks associated to our datasets versus $k$

estimate pruning. For the other baseline, i.e., COMODO we already investigated the efficiency of MinerLSD which showed a considerable reduction in the number of considered patterns, cf., section "Impact of Closed Patterns in Reducing the Search Space". Regarding the number of output patterns, both actually yield the same numbers, if a postprocessing step of COMODO is applied for keeping only the subset of closed patterns (as discussed in section "Similarities and Differences in Pattern Selection"), i.e., by considering all pairs $(c, e)$ with the same (vertex) subgroup $e$ and only keeping the most specific ones. With this postprocessing COMODO returns exactly the same patterns as those output by MinerLSD in our experiments. However, this approach is quite inefficient, cf., section "Impact of Closed Patterns in Reducing the Search Space", since the number of considered patterns is typically considerably larger for COMODO compared to MinerLSD.

Regarding the modularity estimate, we first investigate how the local modularity constraint affects the number of output pairs. In general, as oe(MODL) is an optimistic estimator, we may consider the best possible optimistic estimator which would only develop the \#nec nodes that have at least a descendant $(c, e)$ with local modularity $\operatorname{MODL}(e) \geq l m$. We have then \#lm $\leq \# n e c \leq \# l m e$. Whenever \#lm is far from \#nec this means that there does not exist any good optimistic estimator. Whenever \#lm is close to \#nec which in turn is far from \#lme this means that there could be some optimistic estimator that is much better than oe(MODL). By computing these numbers, we can then state separately for each dataset whether the oe(MODL) estimate is efficient in pruning the search with respect to the best possible estimator nec and whether nec would be efficient in pruning the search, if such an estimator would be found.

Small Datasets In a first step, we first considered several rather small datasets using no minimal support parameters, and a 1-core abstraction in MinerLSD aiming to provide a comparable setting for COMODO. We also checked the number of patterns retrieved by COMODO with additional postprocessing as discussed above - only keeping the closed patterns. We used parameters that do not limit the enumeration in COMODO, i.e., for an exhaustive search only using the local modularity threshold for pruning. Likewise, for MinerLSD, we select and count vertex subgroups whose induced subgraphs satisfy a local 

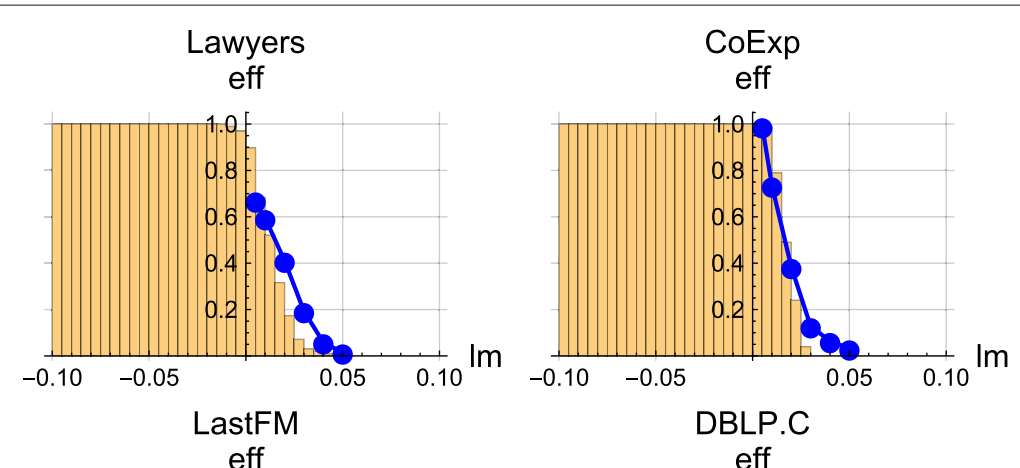

eff
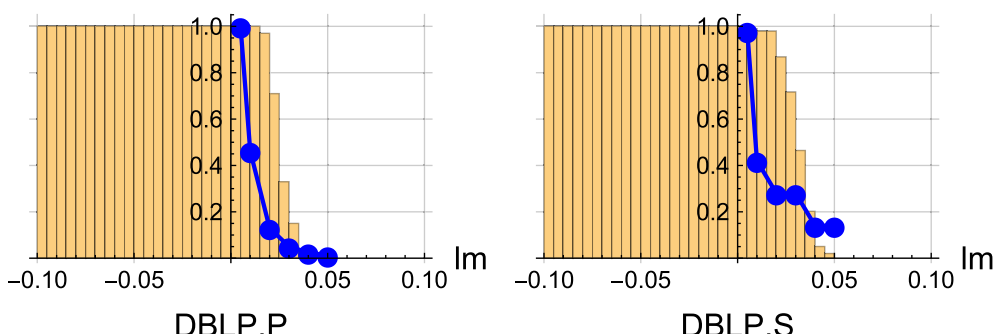

eff
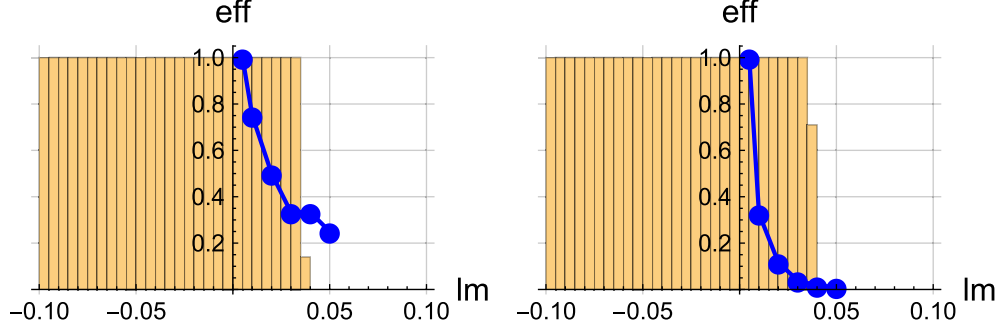

Delicious

eff

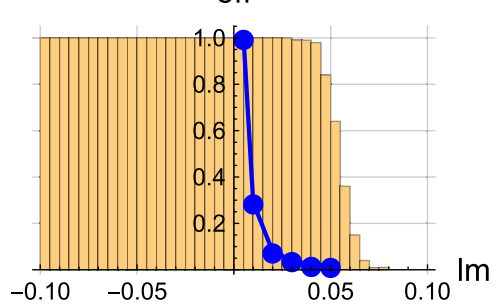

Fig. 3 Detailed Estimated/Observed Modularity Distributions: We consider the unlabeled graph of the dataset. We generate 100 random subgraphs of the unlabelled graph picking randomly half of the vertices. For each random graph, we compute the local modularity of the abstract 5-core subgraph and we report the survival distribution of the local modularity over the 100 experiments (in orange), i.e., for each local modularity (Im) level, the probability of having at least that level in our sample. In blue, we report the (empirically observed) survival distribution of the local modularity, i.e., the respective MODL values of the core subgraphs of the abstract patterns discovered using the 5-core abstraction

modularity threshold $l m$. In this way, we could confirm (again) that the final number of output patterns is the same for both algorithms, as discussed above.

Figure 5 depicts the results of the applied five datasets, with the detailed results in Table 3. Overall, the local modularity estimate is efficient in pruning the pattern exploration, on different levels. For instance, in the Lawyers dataset, MinerLSD finds \#c=3221 patterns at level $l m=0.005$ and most of them, i.e., 2929, have an oe(MODL) value above 0.005 , not too far from the \#nec $=1792$ patterns any top-down search would have to 


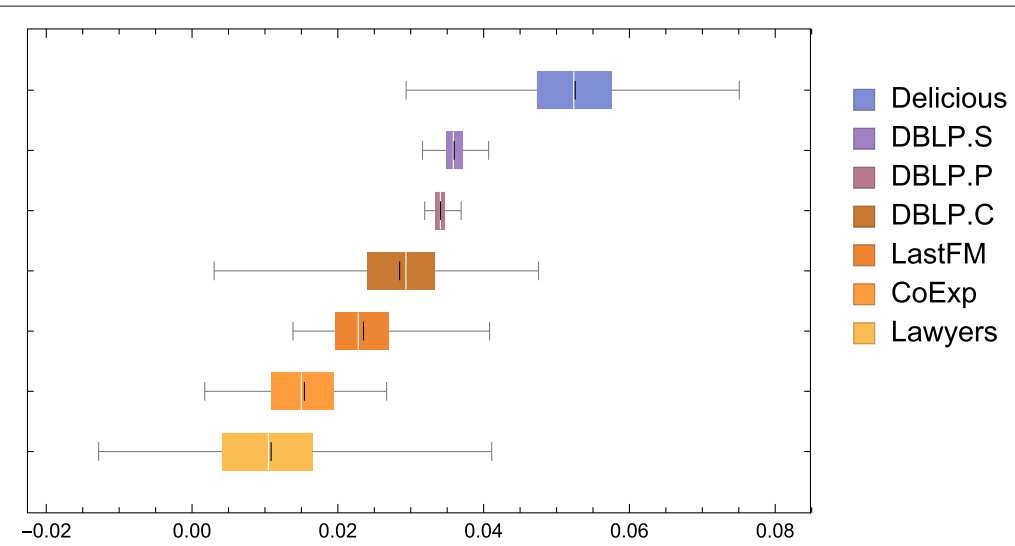

Fig. 4 Distribution of the local modularity of the 5-core abstraction in samples of 100 unlabelled random subgraphs having half of the size (number of vertices) of the original graph

develop anyway to select the 1238 patterns with local modularity MODL above 0.005 . There is then a slow decrease of \#lme while the decrease of \#nec and \#1m is much faster. Yet, pruning does still work, reducing the search effort considerably.

In contrast, for the larger datasets, e.g., for DBLP.P among the \#c $=2396$ patterns only 34 have a local modularity estimate above $0.005,29$ of them have to be developed and 28 do have a local modularity above 0.005 . Furthermore, in the DBLP.C dataset among the $\# \mathrm{c}=14820$ patterns only 179 have a local modularity estimate above $0.005,145$ of them
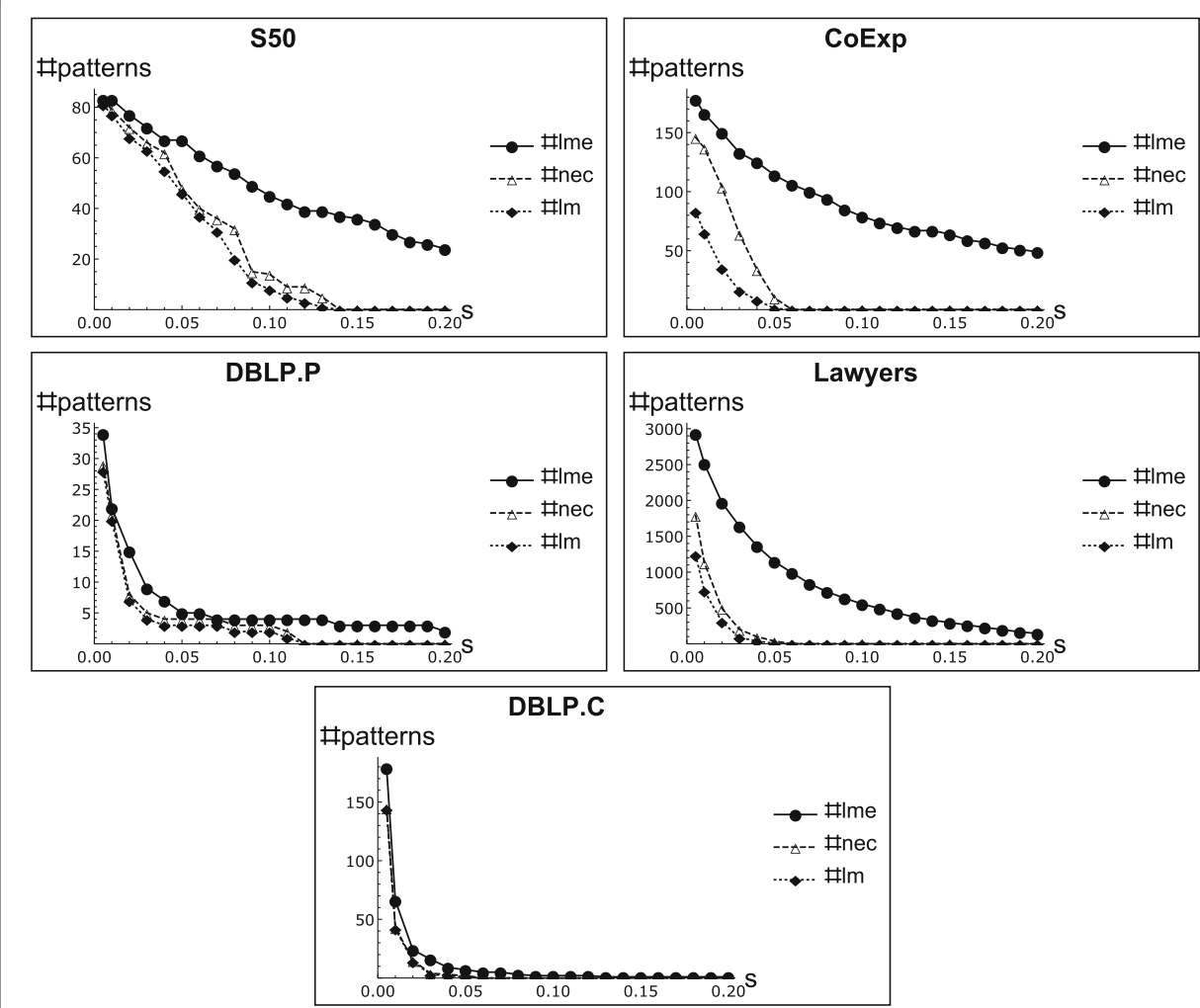

Fig. 5 Numbers of patterns with \#Ime, \#nec and \#Im values (on the Y-axis), above the local modularity threshold (on the X-axis) for 5 attributed networks, using a 1-core abstraction 
Table 3 Number of patterns total, developed, necessary and with required local modularity (according to the respective threshold $0.005 \ldots$... 0.15) using a 1-core abstraction

\begin{tabular}{llllllll}
\hline Data / \#c & $/ 0.005$ & 0.01 & 0.02 & 0.03 & 0.04 & 0.05 & 0.15 \\
\hline S50 & 83 & & & & & & \\
\#Ime & 83 & 83 & 77 & 72 & 67 & 67 & 36 \\
\#nec & 83 & 79 & 72 & 66 & 62 & 48 & 0 \\
\#Im & 81 & 77 & 68 & 63 & 55 & 46 & 0 \\
CoExp & 196 & & & & & & \\
\#Ime & 178 & 166 & 150 & 133 & 125 & 114 & 64 \\
\#nec & 146 & 137 & 104 & 64 & 34 & 10 & 0 \\
\#Im & 83 & 65 & 35 & 16 & 8 & 1 & 0 \\
DBLP.P & 2396 & & & & & & \\
\#Ime & 34 & 22 & 15 & 9 & 7 & 5 & 3 \\
\#nec & 29 & 21 & 8 & 5 & 4 & 4 & 0 \\
\#Im & 28 & 20 & 7 & 4 & 3 & 3 & 0 \\
Lawyers & 3221 & & & & & & \\
\#Ime & 2929 & 2512 & 1970 & 1640 & 1365 & 1146 & 295 \\
\#nec & 1792 & 1131 & 495 & 201 & 99 & 38 & 0 \\
\#Im & 1238 & 738 & 308 & 87 & 39 & 5 & 0 \\
DBLP.C & 14820 & & & & & & \\
\#Ime & 179 & 66 & 24 & 16 & 9 & 7 & 1 \\
\#nec & 145 & 43 & 15 & 4 & 3 & 2 & 0 \\
\#Im & 144 & 42 & 14 & 3 & 2 & 1 & 0 \\
\hline & & & & & & &
\end{tabular}

have to be developed and 144 do have a local modularity above 0.005 . When the local modularity threshold increases, \#lme keeps being close to \#lm.

Overall, the Lawyers dataset displays moderate pruning efficiency, still allowing to avoid to develop many nodes, and this is also the case for the S50 and CoExp datasets. In contrast, DBLP.C and DBLP.P indicate a very efficient optimistic pruning in terms of the numbers of patterns.

Tables 4 and 5 show the runtime results of MinerLSD for the larger of the small datasets (Lawyers, CoExp, DBLP.C, DBLP.P, runtime in seconds). Here, we observe that MinerLSD is either in the same range or slightly faster than MinerLC for the small datasets, i.e., for Lawyers and CoExp. For DBLP.C, we observe a strongly reduced number of patterns, while the runtimes are always in the same range, especially for stronger (graph)constraints. Here, we considered k-cores, $k=1,2,3,5,7$. Therefore, while strongly reducing the number of patterns the additional computation using the estimate still keeps the runtime of the algorithm in the same range as MinerLC most of the times.

In contrast to the other smaller datasets, for the larger DBLP.P dataset we observe an increase in the runtime of MinerLSD compared to MinerLC. However, this can be explained by some special characteristic of DBLP.P. The DBLP.P dataset contains an extremely limited number of labels (32) which are used in the dataset. Here, the extra effort of the estimation does not help too much in decreasing the runtime, because the enumeration in the label space is extremely fast, and hence the check of the patterns is mainly determined by the core abstraction.

Medium Size Datasets Overall, MinerLSD detects closed patterns with the benefit of pruning using the oe(MODL) $\geq \operatorname{lm}$ condition, i.e., only developing the \#lme nodes 
Table 4 MinerLSD \#Im, \#Ime and execution time - small datasets, compared to \#c of MinerLC for same core constraints

\begin{tabular}{|c|c|c|c|c|c|c|c|}
\hline \multirow{2}{*}{$\begin{array}{l}\text { Lawyers } \\
\text { । }\end{array}$} & \multirow{2}{*}{$\begin{array}{l}\text { 1-core } \\
0.005\end{array}$} & \multicolumn{2}{|c|}{$\# c=3221$} & \multicolumn{4}{|c|}{ time $=1$} \\
\hline & & 0.01 & 0.02 & 0.03 & 0.04 & 0.05 & 0.15 \\
\hline \#lme & 2929 & 2512 & 1970 & 1640 & 1365 & 1146 & 295 \\
\hline$\# \mid m$ & 1238 & 738 & 308 & 87 & 39 & 5 & 0 \\
\hline \multirow[t]{2}{*}{ time (s) } & 0 & 1 & 0 & 0 & 0 & 0 & 0 \\
\hline & 2-core & \multicolumn{2}{|c|}{$\# c=2080$} & \multicolumn{4}{|c|}{ time $<1$} \\
\hline \#lme & 2080 & 1938 & 1670 & 1454 & 1265 & 1089 & 291 \\
\hline$\# \mid m$ & 1262 & 775 & 322 & 104 & 41 & 7 & 0 \\
\hline \multirow[t]{2}{*}{ time (s) } & 0 & 0 & 1 & 1 & 0 & 0 & 1 \\
\hline & 3 -core & \multicolumn{2}{|c|}{$\# C=1302$} & \multicolumn{4}{|c|}{ time $<1$} \\
\hline \#lme & 1302 & 1302 & 1215 & 1118 & 1024 & 920 & 282 \\
\hline$\# \mid m$ & 1030 & 746 & 348 & 108 & 43 & 7 & 0 \\
\hline \multirow[t]{2}{*}{ time (s) } & 0 & 0 & 1 & 0 & 0 & 0 & 0 \\
\hline & 5-core & \multicolumn{2}{|c|}{$\# c=463$} & \multicolumn{4}{|c|}{ time $<1$} \\
\hline \#lme & 463 & 463 & 463 & 459 & 449 & 432 & 202 \\
\hline$\# \mid m$ & 413 & 366 & 253 & 119 & 36 & 9 & 0 \\
\hline \multirow[t]{2}{*}{ time (s) } & 0 & 0 & 1 & 1 & 0 & 0 & 0 \\
\hline & 7-core & \multicolumn{2}{|c|}{$\# C=155$} & \multicolumn{4}{|c|}{ time $<1$} \\
\hline \#lme & 155 & 155 & 155 & 155 & 155 & 155 & 115 \\
\hline$\# \mid m$ & 147 & 133 & 97 & 62 & 36 & 13 & 0 \\
\hline time (s) & 1 & 1 & 0 & 0 & 0 & 1 & 0 \\
\hline CoExp & 1-core & $\# C=1$ & & time & & & \\
\hline I & 0.005 & 0.01 & 0.02 & 0.03 & 0.04 & 0.05 & 0.15 \\
\hline \#lme & 178 & 166 & 150 & 133 & 125 & 114 & 64 \\
\hline$\# \mid m$ & 83 & 65 & 35 & 16 & 8 & 1 & 0 \\
\hline \multirow[t]{2}{*}{ time (s) } & 0 & 0 & 0 & 0 & 0 & 0 & 1 \\
\hline & 2-core & \multicolumn{2}{|c|}{$\# C=172$} & \multicolumn{4}{|c|}{ time $<1$} \\
\hline \#lme & 162 & 153 & 141 & 125 & 118 & 108 & 64 \\
\hline$\# \mid m$ & 89 & 78 & 51 & 26 & 12 & 3 & 0 \\
\hline \multirow[t]{2}{*}{ time (s) } & 0 & 0 & 0 & 1 & 0 & 0 & 0 \\
\hline & 3 -core & \multicolumn{2}{|c|}{$\# C=138$} & \multicolumn{4}{|c|}{ time $<1$} \\
\hline \#lme & 134 & 129 & 118 & 109 & 102 & 95 & 56 \\
\hline$\# \mid m$ & 75 & 64 & 42 & 23 & 12 & 0 & 0 \\
\hline \multirow[t]{2}{*}{ time (s) } & 0 & 0 & 0 & 0 & 0 & 0 & 0 \\
\hline & 5-core & \multicolumn{2}{|c|}{$\# c=62$} & time & & & \\
\hline \#lme & 62 & 60 & 57 & 51 & 48 & 47 & 31 \\
\hline$\# \mid m$ & 31 & 23 & 12 & 4 & 2 & 1 & 0 \\
\hline time (s) & 1 & 0 & 0 & 0 & 1 & 0 & 0 \\
\hline & 7-core & $\# c=3$ & & time & & & \\
\hline \#lme & 37 & 37 & 36 & 34 & 33 & 32 & 19 \\
\hline$\# \mid m$ & 27 & 22 & 17 & 5 & 3 & 2 & 0 \\
\hline time (s) & 0 & 0 & 0 & 0 & 0 & 0 & 0 \\
\hline
\end{tabular}

according to Table 2. Furthermore, applying both the k-cores and local modularity constraints makes it possible to find some balance between the k-core and the local modularity constraint to apply when facing large datasets that are difficult to mine. This is investigated on the two datasets LastFM and Delicious, i.e., those with the largest number of closed core patterns when considering the 1-core and no local modularity thresholds 
Table 5 MinerLSD \#Im, \#Ime and execution time - DBLP.C and DBLP.P, compared to \#C of MinerLC for same core constraints

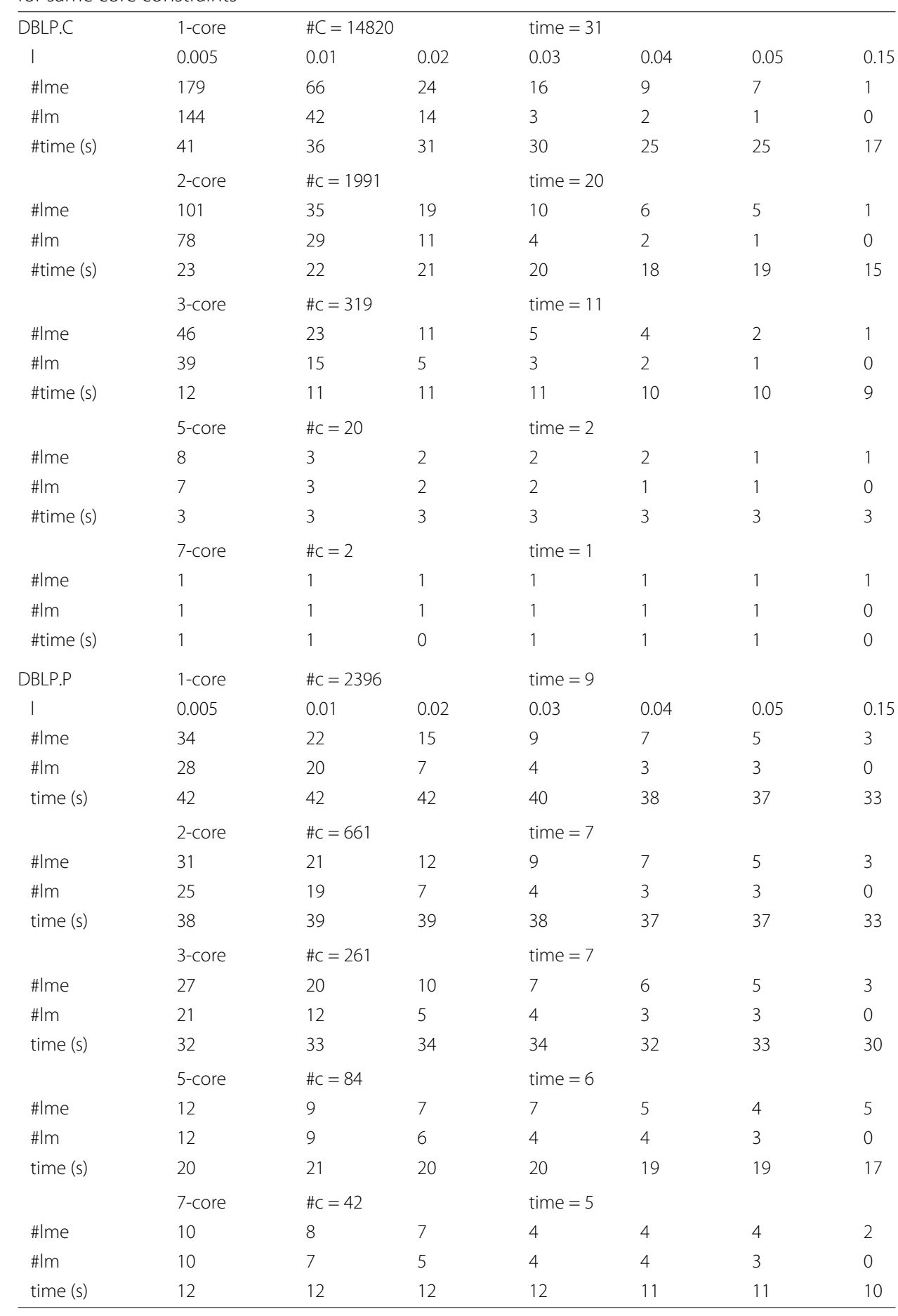

- these were not investigated in Tables 2 and 3, respectively. For these medium sized datasets, we performed experiments using 1-cores, 2-cores, 3-cores, 5-cores and 7-cores with local modularity thresholds $0.01,0.02,0.03,0.04,0.05$, and 0.15 ; the results regarding the number of closed patterns and the total CPU time (including pruning/optimistic estimation) are shown in Fig. 6 (runtimes in seconds). 


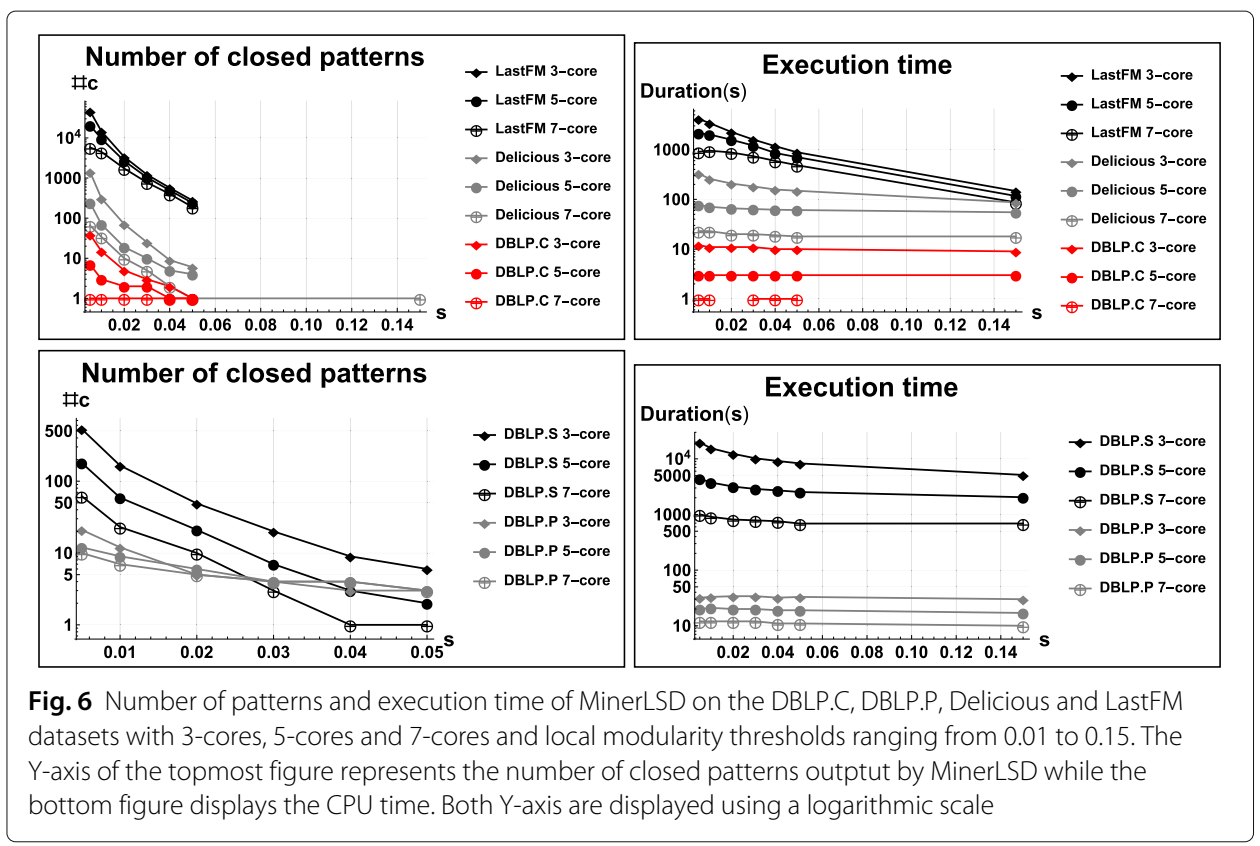

The benefit of applying local modularity constraints in the resulting number of closed patterns is, as expected, quite impressive. When no constraint (outside the 1-core) is applied, MinerLC in comparison finds 1,555,292 and 11,833,577 closed patterns, respectively. For MinerLSD, in the LastFM case there are no strong differences when using 1-cores, 2-cores and 3-cores while we know from Fig. 2 that using 4-cores does have an important effect. Corresponding results are also observed for larger sizes of the respective k-cores. Regarding the Delicious dataset, we observe a smaller number of patterns at local modularity levels 0.04 and 0.05 with 1 -cores than with 2 and 3-cores. When no local modularity constraint is applied the closed patterns with 2 and 3-cores are a subset of the closed patterns with 1-cores, therefore the results seem counterintuitive at first. However, for the same pattern the 3-core subgraph is smaller than the 1-core subgraph and may have better local modularity, which happens in the Delicious case.

Regarding the CPU times, we observe a considerable decrease using appropriate local modularity thresholds for both LastFM and Delicious which is especially important for weaker (graph-)constraints, i.e., with respect to the applied $k$-cores. Using the appropriate modularity thresholds the runtime can be considerably decreased which enables new approaches already for medium sized datasets, e.g., concerning pattern exploration. Specifically, if we compare the extra computation performed by MinerLSD for computing the estimate, in the Delicious case, the benefit is immediately obvious: MinerLSD is always much faster than MinerLC. The LastFM dataset shows a somewhat different picture: with weaker core-constraints and at local modularity level of 0.01 MinerLC (which does not consider local modularity) is (slightly) faster than MinerLSD. This is not that surprising, since MinerLSD has to compute local modularity estimates and local modularities for all the developed patterns during search. However, first this happens only for weak constraints, and second, when using MinerLC all these computations (in fact much more as there is no pruning), would have to be made anyway in post-processing fashion for obtaining the patterns according to a local modularity threshold. Furthermore, the 
runtime behavior of LastFM here is similar to DBLP.P and can also be explained by the smaller number of labels compared to Delicious. Overall, this shows that if we consider appropriate local modularity thresholds MinerLSD already allows the analysis of larger datasets, especially in terms of larger sizes of the labels, while comparable results (with respect to MinerLC) are usually obtained for weak (graph-)constraints. However, the efficient pruning of MinerLSD is important, e.g., for exploration, and also for the processing of larger datasets, as we will also discuss in the next section for large datasets. Detailed results are presented in Table 6 which also displays the \#lme numbers.

Large Datasets In this section, we present experiments of MinerLSD on two large datasets, namely DBLP.S and DBLP.XL (see Table 1 for their characteristics) to further explore the scalability of MinerLSD when using both $k$-core and local modularity constraints. Again we do not use any threshold on the pattern supports.

In Table 7, we report the results on DBLP.S and DBLP.XL with the same local modularity thresholds as in the previous section and applying $k=1,2,3,5,7$ and $7 \mathrm{k}$-core constraints, respectively. The scalability of MinerLSD depends obviously on the size and density of the network but also heavily depends on the size of the attribute set and on the average number of labels per vertex. DBLP.XL is then a real challenge as it is a large network made of 929,937 vertices related by 3,461,697 edges and described by more than 90,000 items, with an average number of 10.16 labels per vertex. The efficiency of the optimistic pruning is then of primary importance.

As can be seen in the results table, optimistic estimate pruning using local modularity is quite effective in achieving an efficient pattern mining approach. For both datasets, we observe large reductions in the number of patterns, while focussing on the interesting ones according to the applied local modularity interestingness measure and the utilized local modularity thresholds. In particular, the results for DBLP.S indicate the enormous pruning efficiency - here the dataset for weaker constraints cannot be handled by MinerLC at all, where the computation did not terminate after $36 \mathrm{~h}$. The DBLP.XL results indicate the same trend. Overall, this indicates the huge impact of optimistic estimate pruning using local modularity as provided by MinerLSD for handling large datasets.

\section{Structural Pattern Set Analysis}

In the following, we analyze the results of the proposed pattern mining method Miner $L S D$ in more detail, focussing on different graph statistics. We report exemplary results on three datasets with different characteristics as outlined in section "Datasets", i.e., the Lawyers, the CoExp, and the DBLP.C datasets. We consider all patterns above a given local modularity threshold, combined with different core abstractions. For computing the graph statistics, we analyze the respective induced subgraph $W$ of each pattern, and consider the following: (1) the vertex count $N_{W},(2)$ the edge count $E_{W}$, (3) the scaled density (cf., (Lancichinetti et al. 2010)) of subgraph $W$, i.e., the ratio of $E_{W}$ divided by the number of edges of a complete graph with the same number of vertices as $W$ and multiplied (scaled) by the total number of vertices; this measure approximately estimates the average degree of the nodes contained in the community, cf., (Lancichinetti et al. 2010). (4) Furthermore, we also consider the fraction of outgoing edges, i.e., the edges connecting nodes contained in the pattern with others not being part of the pattern subgraph, to the set of edges $E_{W}$. The results are shown in Tables 8, 9, 10 and 11 . 
Table 6 MinerLSD \#Im, \#Ime and execution time compared to \#c of MinerLC for same core constraints

\begin{tabular}{|c|c|c|c|c|c|c|c|}
\hline \multirow{2}{*}{$\begin{array}{l}\text { LastFM } \\
1\end{array}$} & \multirow{2}{*}{$\begin{array}{l}1 \text {-core } \\
0.005\end{array}$} & \multicolumn{2}{|c|}{$\# C=1555292$} & \multicolumn{4}{|c|}{ time $=2874$} \\
\hline & & 0.01 & 0.02 & 0.03 & 0.04 & 0.05 & 0.15 \\
\hline \#lme & & 59528 & 16163 & 6817 & 3475 & 1920 & 52 \\
\hline$\# \mid m$ & & 17627 & 3633 & 1238 & 575 & 276 & 0 \\
\hline \multirow[t]{2}{*}{ time (s) } & & 5816 & 3400 & 2252 & 1605 & 1187 & 196 \\
\hline & 2-core & \multicolumn{2}{|c|}{$\# C=471546$} & \multicolumn{4}{|c|}{ time $=2320$} \\
\hline I & 0.005 & 0.01 & 0.02 & 0.03 & 0.04 & 0.05 & 0.15 \\
\hline \#lme & & 50507 & 14752 & 6464 & 3349 & 1856 & 52 \\
\hline$\# \mid m$ & & 16751 & 3646 & 1252 & 583 & 282 & 0 \\
\hline \multirow[t]{2}{*}{ time (s) } & & 4668 & 2915 & 1995 & 1452 & 1073 & 178 \\
\hline & 3-core & \multicolumn{2}{|c|}{$\# C=161764$} & \multicolumn{4}{|c|}{ time $=1878$} \\
\hline I & 0.005 & 0.01 & 0.02 & 0.03 & 0.04 & 0.05 & 0.15 \\
\hline \#lme & 87211 & 39127 & 12694 & 5753 & 3039 & 1720 & 50 \\
\hline$\# \mid m$ & 46400 & 14637 & 3377 & 1219 & 572 & 276 & 0 \\
\hline \multirow[t]{2}{*}{ time (s) } & 4149 & 3422 & 2262 & 1596 & 1174 & 885 & 147 \\
\hline & 5-core & \multicolumn{2}{|c|}{$\# C=26312$} & \multicolumn{4}{|c|}{ time $=1069$} \\
\hline I & 0.005 & 0.01 & 0.02 & 0.03 & 0.04 & 0.05 & 0.15 \\
\hline \#lme & 24807 & 18103 & 8224 & 4272 & 2352 & 1412 & 46 \\
\hline$\# 1 \mathrm{~m}$ & 20562 & 9507 & 2680 & 1035 & 496 & 239 & 0 \\
\hline \multirow[t]{2}{*}{ time (s) } & 2148 & 2013 & 1580 & 1206 & 857 & 706 & 117 \\
\hline & 7-core & \multicolumn{2}{|c|}{$\# c=5859$} & \multicolumn{4}{|c|}{ time $=531$} \\
\hline I & 0.005 & 0.01 & 0.02 & 0.03 & 0.04 & 0.05 & 0.15 \\
\hline \#lme & 5854 & 5620 & 4031 & 2533 & 1517 & 994 & 39 \\
\hline$\# \mid m$ & 5814 & 4482 & 1737 & 775 & 402 & 189 & 0 \\
\hline time (s) & 902 & 953 & 877 & 738 & 594 & 486 & 87 \\
\hline Delicious & 1-core & \multicolumn{2}{|c|}{$\# C=11833577$} & \multicolumn{4}{|c|}{ time $=121934$} \\
\hline I & 0.005 & 0.01 & 0.02 & 0.03 & 0.04 & 0.05 & 0.15 \\
\hline \#lme & & 5655 & 776 & 255 & 121 & 71 & 4 \\
\hline$\# 1 \mathrm{~m}$ & & 2214 & 165 & 31 & 6 & 1 & 0 \\
\hline \multirow[t]{2}{*}{ time (s) } & & 5296 & 2018 & 1173 & 825 & 643 & 179 \\
\hline & 2-core & \multicolumn{2}{|c|}{$\# C=130458$} & time $=$ & & & \\
\hline । & 0.005 & 0.01 & 0.02 & 0.03 & 0.04 & 0.05 & 0.15 \\
\hline \#lme & 7251 & 1421 & 288 & 116 & 65 & 37 & 3 \\
\hline$\# \mid m$ & 5440 & 879 & 138 & 39 & 11 & 6 & 0 \\
\hline time (s) & 1499 & 920 & 569 & 426 & 358 & 298 & 129 \\
\hline & 3-core & $\# C=11$ & & time $=$ & & & \\
\hline I & 0.005 & 0.01 & 0.02 & 0.03 & 0.04 & 0.05 & 0.15 \\
\hline \#lme & 1729 & 430 & 114 & 51 & 25 & 17 & 1 \\
\hline$\# \mid \mathrm{m}$ & 1419 & 311 & 71 & 25 & 9 & 6 & 0 \\
\hline time (s) & 331 & 259 & 208 & 182 & 158 & 149 & 87 \\
\hline & 5-core & $\# C=57$ & & time $=$ & & & \\
\hline I & 0.005 & 0.01 & 0.02 & 0.03 & 0.04 & 0.05 & 0.15 \\
\hline \#lme & 296 & 89 & 25 & 14 & 7 & 6 & 1 \\
\hline$\# \mid m$ & 241 & 70 & 19 & 10 & 5 & 4 & 0 \\
\hline time (s) & 77 & 71 & 66 & 64 & 62 & 61 & 55 \\
\hline & 7-core & $\# C=77$ & & time $=$ & & & \\
\hline I & 0.005 & 0.01 & 0.02 & 0.03 & 0.04 & 0.05 & 0.15 \\
\hline \#lme & 67 & 41 & 13 & 7 & 4 & 1 & 1 \\
\hline$\# \mid m$ & 66 & 34 & 10 & 5 & 2 & 1 & 1 \\
\hline time (s) & 23 & 23 & 20 & 20 & 19 & 18 & 18 \\
\hline
\end{tabular}


Table 7 MinerLSD \#Im, \#Ime and execution time compared to \#c of MinerLC for same core constraints

\begin{tabular}{|c|c|c|c|c|c|c|c|}
\hline \multirow{2}{*}{$\begin{array}{c}\text { DBLP.S } \\
\text { । }\end{array}$} & \multirow{2}{*}{$\begin{array}{l}1 \text {-core } \\
0.005\end{array}$} & \multicolumn{2}{|c|}{$\# c \geq 3457143$} & \multicolumn{4}{|c|}{ time $=$ STOPPED AFTER 36h } \\
\hline & & 0.01 & 0.02 & 0.03 & 0.04 & 0.05 & 0.15 \\
\hline \#lme & 1150 & 351 & 103 & 50 & 26 & 18 & 1 \\
\hline$\# \mid m$ & 778 & 230 & 68 & 25 & 12 & 6 & 0 \\
\hline \multirow[t]{2}{*}{ time (s) } & 59989 & 37645 & 24906 & 20634 & 17299 & 16167 & 8332 \\
\hline & 2-core & \multicolumn{2}{|c|}{$\# C \geq 3584834$} & \multicolumn{3}{|c|}{ time $=$ STOPPED AFTER 36h } & \\
\hline I & 0.005 & 0.01 & 0.02 & 0.03 & 0.04 & 0.05 & 0.15 \\
\hline \#lme & 958 & 303 & 94 & 44 & 24 & 16 & 1 \\
\hline$\# \mid m$ & 722 & 218 & 64 & 24 & 12 & 6 & 0 \\
\hline \multirow[t]{2}{*}{ time (s) } & 36302 & 25949 & 19065 & 16068 & 13869 & 12907 & 7073 \\
\hline & 3-core & \multicolumn{2}{|c|}{$\# C=1576164$} & \multicolumn{3}{|c|}{ time $=45720$} & \\
\hline I & 0.005 & 0.01 & 0.02 & 0.03 & 0.04 & 0.05 & 0.15 \\
\hline \#lme & 621 & 208 & 72 & 28 & 17 & 9 & 1 \\
\hline$\# \mid m$ & 533 & 165 & 49 & 20 & 9 & 6 & 0 \\
\hline \multirow[t]{2}{*}{ time (s) } & 19799 & 15531 & 12329 & 10221 & 9149 & 8276 & 5143 \\
\hline & 5 -core & \multicolumn{2}{|c|}{$\# C=44345$} & \multicolumn{3}{|c|}{ time $=3791$} & \\
\hline I & 0.005 & 0.01 & 0.02 & 0.03 & 0.04 & 0.05 & 0.15 \\
\hline \#lme & 200 & 71 & 26 & 10 & 6 & 4 & 1 \\
\hline$\# \mid m$ & 180 & 59 & 21 & 7 & 3 & 2 & 0 \\
\hline \multirow[t]{2}{*}{ time (s) } & 4410 & 3760 & 3173 & 2877 & 2709 & 2533 & 2044 \\
\hline & 7-core & \multicolumn{2}{|c|}{$\# c=5659$} & \multicolumn{3}{|c|}{ time $=881$} & \\
\hline I & 0.005 & 0.01 & 0.02 & 0.03 & 0.04 & 0.05 & 0.15 \\
\hline \#lme & 62 & 24 & 10 & 4 & 2 & 1 & 1 \\
\hline$\# \mid m$ & 62 & 23 & $10 c$ & 3 & 1 & 1 & 0 \\
\hline time (s) & 1005 & 908 & 812 & 784 & 756 & 687 & 689 \\
\hline DBLP.XL & 7-core & \multicolumn{2}{|c|}{$\# c=9206$} & \multicolumn{3}{|c|}{ time $=93906$} & \\
\hline । & 0.005 & 0.01 & 0.02 & 0.03 & 0.04 & 0.05 & 0.15 \\
\hline \#lme & 10 & 5 & 4 & 3 & 2 & 1 & 1 \\
\hline$\# \mid m$ & 9 & 5 & 3 & 1 & 1 & 1 & 0 \\
\hline time (s) & 113790 & 111079 & 110142 & 107967 & 103363 & 97326 & 96811 \\
\hline
\end{tabular}

Considering the results shown in Tables 8 and 9 we observe that, as expected, increasing numbers of $k$ tend to focus on larger communities, which is especially the case for weaker core constraints and larger local modularity thresholds. In particular, we observe those trends for the local modularity for the Lawyers and the DBLP.C datasets, while this is also pronounced for CoExp regarding stronger constraints. For the DBLP.C network, in particular, we observe a rather strong effect. Overall, with no constraints quite small patterns are detected. When the $k$-core constraint and the local modularity threshold are increased, then larger patterns are detected which are also considerably denser than those with no constraints. This can clearly be observed in Table 10 for increasing $k$-core and local modularity threshold values. Furthermore, when we consider the ratio of outgoing edges vs. in-edges of a pattern shown in Table 11, then we also observe the trend that the proposed approach focuses on selecting denser pattern subgraphs with a stronger connectivity structure in terms of the links within the subgraph, i.e., the in-edges. This is especially obvious for higher $k$-core and local modularity threshold values, as exemplified by the CoExp and DBLP.C datasets, e.g., for $k=5$ and $l m=0.04$ where the number of in-edges strongly "dominates" the number of outgoing edges. 
Table 8 Vertex Counts: Mean and standard deviation (in brackets) of the number of vertices of the pattern support, i.e., of the induced pattern subgraphs, for different values of $k$ and the local modularity threshold $/ \mathrm{m}$

\begin{tabular}{llllll}
\hline $\mathrm{k}$ & No $I \mathrm{~m}$ & $\mathrm{Im}=0.005$ & $\mathrm{Im}=0.01$ & $\mathrm{Im}=0.02$ & $\mathrm{Im}=0.04$ \\
\hline \multicolumn{2}{l}{ Lawyers: $n=71 \mathrm{~m}=556$} & & & & \\
1 & $12.7(9.6)$ & $17.2(9.5)$ & $20.11(9.6)$ & $24.4(9.5)$ & $29.8(4.59)$ \\
2 & $15.3(10.0)$ & $16.8(9.4)$ & $19.35(9.3)$ & $23.4(9.2)$ & $27.7(5.6)$ \\
3 & $18.0(10.6)$ & $18.0(9.5)$ & $19.49(9.2)$ & $22.9(9.0)$ & $26.8(6.3)$ \\
5 & $23.2(11.9)$ & $21.7(10.1)$ & $21.50(9.0)$ & $23.7(8.3)$ & $27.3(5.6)$ \\
CoExp: $n=151, m=1849$ & & & & \\
1 & $59.5(44.0)$ & $51.9(35.8)$ & $51.5(32.8)$ & $54.6(31.6)$ & $53.9(21.9)$ \\
2 & $56.9(41.5)$ & $49.2(31.9)$ & $51.9(29.4)$ & $57.2(25.9)$ & $63.0(18.5)$ \\
3 & $54.7(37.9)$ & $47.2(31.6)$ & $47.4(28.8)$ & $52.2(24.7)$ & $60.2(18.3)$ \\
5 & $50.0(36.2)$ & $48.3(34.2)$ & $51.6(33.1)$ & $54.9(28.9)$ & $85.5(9.2)$ \\
DBLP.C: $n=3140, m=10689$ & & & & \\
1 & $8.3(30.8)$ & $124.0(106.1)$ & $231.3(144.0)$ & $373.9(163.6)$ & $631.0(63.6)$ \\
2 & $12.5(68.3)$ & $124.6(325.9)$ & $159.5(103.9)$ & $248.0(119.0)$ & $434.5(64.4)$ \\
3 & $21.7(126.4)$ & $117.3(350.3)$ & $250.7(549.6)$ & $604.6(906.4)$ & $1256.5(1366.8)$ \\
5 & $62.6(199.6)$ & $164.9(327.7)$ & $351.3(480.6)$ & $511.0(555.8)$ & $904.0(0.0)$ \\
\hline
\end{tabular}

\section{Pattern Selection and K-Core Abstraction}

In this section, we provide examples of patterns demonstrating the benefits of pattern selection using local modularity and k-core abstraction. In particular, we discuss illustrative examples from two different datasets - the Lawyers and the (larger) DBLP.C dataset.

Lawyers Dataset In order to demonstrate the effectiveness of the pattern exploration and selection methodology using abstract closed pattern with k-cores and local modularity, we exemplify that with the two patterns shown in Fig. 7. Here, we show two similar patterns in terms of Jaccard similarity (0.52) considering the nodes of the respective pattern-induced subgraphs. While the patterns are very similar regarding the overlap

Table 9 Edge Counts: Mean and standard deviation (in brackets) of the number of edges of the pattern support, i.e., of the induced pattern subgraphs, for different values of $k$ and the local modularity threshold $/ \mathrm{m}$

\begin{tabular}{clllll}
\hline $\mathrm{k}$ & No $\mathrm{Im}$ & $\mathrm{Im}=0.005$ & $\mathrm{Im}=0.01$ & $\mathrm{Im}=0.02$ & $\mathrm{Im}=0.04$ \\
\hline \multicolumn{2}{c}{ Lawyers: $n=71 \mathrm{~m}=556$} & & & & \\
1 & $38.2(58.0)$ & $61.4(61.4)$ & $78.4(64.0)$ & $103.2(67.1)$ & $25.6(63.9)$ \\
2 & $53.8(66.3)$ & $62.7(61.9)$ & $78.3(64.4)$ & $104.1(67.3)$ & $126.1(68.1)$ \\
3 & $73.2(75.4)$ & $72.9(65.5)$ & $82.2(64.4)$ & $104.5(65.0)$ & $124.8(67.9)$ \\
5 & $121.7(95.5)$ & $109.9(77.0)$ & $108.2(67.7)$ & $123.7(62.6)$ & $135.7(59.8)$ \\
CoExp: $n=151, m=1849$ & & & & \\
1 & $365.4(473.9)$ & $323.1(512.8)$ & $327.1(497.0)$ & $392.0(579.7)$ & $448.6(653.1)$ \\
2 & $404.7(488.8)$ & $317.2(492.9)$ & $325.0(483.7)$ & $352.2(492.9)$ & $377.4(529.4)$ \\
3 & $445.1(496.5)$ & $385.1(535.0)$ & $375.7(525.1)$ & $394.6(529.5)$ & $481.5(598.9)$ \\
5 & $525.4(499.6)$ & $551.8(563.1)$ & $625.0(548.9)$ & $729.3(544.9)$ & $1495.0(132.9)$ \\
DBLP.C: $n=3140, m=10689$ & & & \\
1 & $7.4(91.4)$ & $164.9(192.5)$ & $347.3(282.5)$ & $627.8(340.3)$ & $1278.0(356.4)$ \\
2 & $22.8(240.5)$ & $316.81(1183.3)$ & $349.2(271.3)$ & $577.7(328.6)$ & $1126.0(357.8)$ \\
3 & $66.7(531.01)$ & $419.7(1487.5)$ & $945.3(2350.3)$ & $2393.0(3924.2)$ & $5229.0(5897.3)$ \\
5 & $347.3(1246.9)$ & $950.4(2066.5)$ & $2101.3(3055.2)$ & $3085.0(3586.5)$ & $5621.0(0.0)$ \\
\hline
\end{tabular}


Table 10 Scaled Graph Densities: Mean and standard deviation (in brackets) of the scaled densities of the pattern support, i.e., of the induced pattern subgraphs, for different values of $k$ and the local modularity threshold $/ \mathrm{m}$

\begin{tabular}{ccllll}
\hline $\mathrm{k}$ & No $I \mathrm{~m}$ & $\mathrm{Im}=0.005$ & $\mathrm{Im}=0.01$ & $\mathrm{Im}=0.02$ & $\mathrm{Im}=0.04$ \\
\hline \multicolumn{2}{l}{ Lawyers: $n=71 \mathrm{~m}=556$} & & & & \\
1 & $4.59(2.63)$ & $6.34(2.51)$ & $7.26(2.46)$ & $8.13(2.61)$ & $8.98(3.27)$ \\
2 & $5.92(2.56)$ & $6.63(2.47)$ & $7.50(2.38)$ & $8.50(2.42)$ & $9.43(2.82)$ \\
3 & $7.23(2.47)$ & $7.42(2.32)$ & $7.92(2.27)$ & $8.80(2.27)$ & $9.45(2.67)$ \\
5 & $9.89(2.28)$ & $9.74(2.10)$ & $9.82(1.96)$ & $10.38(1.80)$ & $11.38(1.95)$ \\
7 & $12.23(2.08)$ & $12.15(2.05)$ & $11.99(1.86)$ & $12.20(1.40)$ & $13.14(1.20)$ \\
CoExp: $n=151, m=1849$ & & & & $12.19(13.90)$ \\
1 & $9.58(8.34)$ & $9.38(9.54)$ & $10.14(9.83)$ & $11.17(11.12)$ & $10.19(11.46)$ \\
2 & $11.15(8.37)$ & $9.78(9.09)$ & $10.14(9.18)$ & $10.41(9.85)$ & $13.22(13.20)$ \\
3 & $13.07(8.60)$ & $12.02(9.66)$ & $12.15(9.83)$ & $12.22(10.44)$ & $35.42(0.71)$ \\
5 & $17.92(8.25)$ & $18.40(9.00)$ & $20.68(8.50)$ & $25.22(5.72)$ & \\
DBLP.C: $n=3140, m=10689$ & & & & $4.02(0.73)$ \\
1 & $1.73(0.57)$ & $2.60(0.82)$ & $2.93(0.75)$ & $3.38(0.65)$ & $5.13(0.89)$ \\
2 & $3.15(0.68)$ & $4.15(0.98)$ & $4.33(0.72)$ & $4.66(0.68)$ & $12.89(0.80)$ \\
3 & $4.73(0.87)$ & $5.90(0.78)$ & $6.35(0.79)$ & $6.74(1.14)$ & \\
5 & $7.30(1.57)$ & $8.55(1.94)$ & $10.16(2.02)$ & $10.92(2.17)$ & \\
\hline
\end{tabular}

and their size, they have quite different local modularity values referring to their connectivity structure. The left pattern described by $35<$ Age $\leq 65$ AND Seniority $<5$ AND Status $=$ Partner, with a size $=24$ of the set of nodes in its subgraph, is considerably denser with a local modularity of MODL $=0.058$, compared to the pattern on the right; the latter is described by Age $<40$ AND Seniority $\leq 30$, with a size $=23$ of the pattern support and a local modularity of only MODL $=0.013$. Therefore, while both patterns are abstract closed patterns according to similar support criteria and the 5-core abstraction, a higher modularity threshold, e.g., MODL $\geq 0.05$ would only select the first (left pattern in Fig. 7) instead of the right pattern. From the description, we can also observe that the

Table 11 Ratio of outgoing edges to edges in the pattern subgraph (in-edges): Mean and standard deviation (in brackets) of that ratio of the pattern support, i.e., of the induced pattern subgraphs, for different values of $k$ and the local modularity threshold $/ m$

\begin{tabular}{cccccc}
\hline $\mathrm{k}$ & No $I \mathrm{~m}$ & $\mathrm{Im}=0.005$ & $\mathrm{Im}=0.01$ & $I \mathrm{~m}=0.02$ & $\mathrm{Im}=0.04$ \\
\hline \multicolumn{2}{l}{ Lawyers: $n=71 \mathrm{~m}=556$} & & & & \\
1 & $10.99(9.07)$ & $4.90(2.55)$ & $3.68(1.72)$ & $2.68(1.19)$ & $1.80(0.65)$ \\
2 & $6.58(4.40)$ & $4.81(2.53)$ & $3.67(1.72)$ & $2.64(1.16)$ & $1.81(0.65)$ \\
3 & $4.62(2.81)$ & $4.14(2.18)$ & $3.46(1.58)$ & $2.58(1.10)$ & $1.84(0.66)$ \\
5 & $2.74(1.62)$ & $2.82(1.53)$ & $2.69(1.32)$ & $2.19(0.89)$ & $1.56(0.52)$ \\
CoExp: $n=151, m=1849$ & & & & \\
1 & $4.95(8.72)$ & $2.46(1.59)$ & $2.13(1.36)$ & $1.62(1.11)$ & $0.69(0.34)$ \\
2 & $3.88(4.76)$ & $2.69(1.79)$ & $2.48(1.52)$ & $2.24(1.35)$ & $1.69(1.10)$ \\
3 & $3.58(4.48)$ & $2.83(2.33)$ & $2.58(1.77)$ & $2.41(1.49)$ & $1.82(1.14)$ \\
5 & $3.75(4.12)$ & $2.89(2.75)$ & $2.24(2.04)$ & $1.57(1.07)$ & $0.16(0.10)$ \\
DBLP.C: $n=3140, m=10689$ & & & & \\
1 & $14.23(13.72)$ & $6.85(1.94)$ & $5.28(1.19)$ & $3.94(0.80)$ & $2.39(0.41)$ \\
2 & $11.29(10.37)$ & $5.99(2.09)$ & $4.88(1.17)$ & $3.78(0.77)$ & $2.44(0.50)$ \\
3 & $11.44(8.04)$ & $5.96(1.90)$ & $4.57(1.64)$ & $2.96(1.82)$ & $1.27(1.68)$ \\
5 & $10.99(8.22)$ & $5.99(3.07)$ & $3.13(2.53)$ & $1.99(2.22)$ & $0.42(0)$ \\
\hline
\end{tabular}




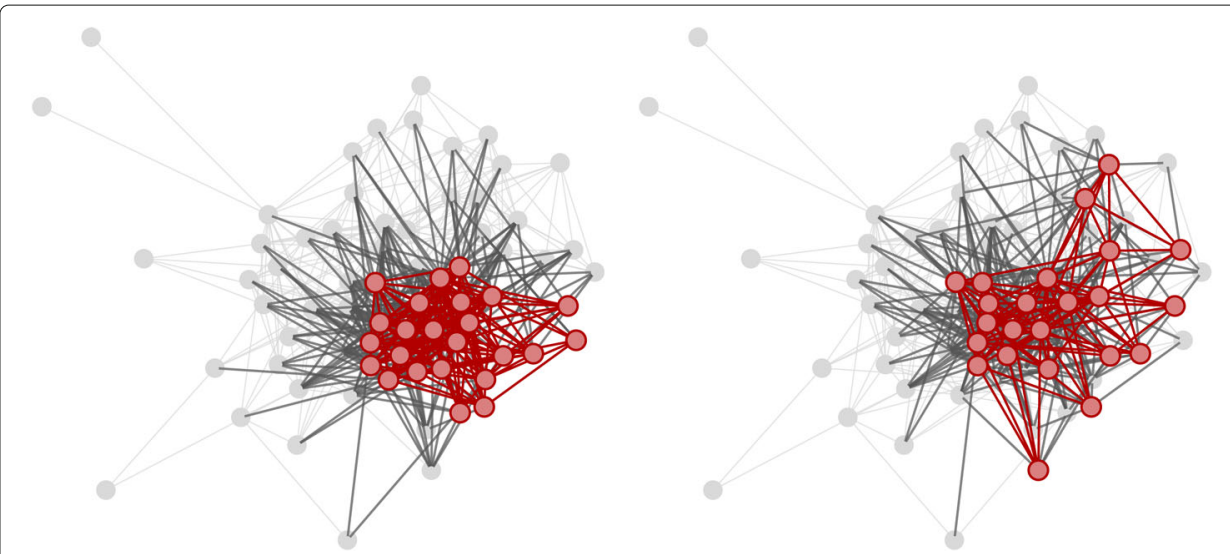

Fig. 7 Example patterns from the Lawyers dataset: Both patterns are similar 5-cores, with a Jaccard similarity considering the nodes of the respective pattern-induced subgraphs of 0.52 . The pattern on the left (described by $35<$ Age $\leq 65$ AND Seniority $<5$ AND Status = Partner, with size $=24$ ) is considerably denser with a local modularity of $M O D L=0.058$, compared to the pattern on the right (described by Age $<40$ AND Seniority $\leq 30$, with size $=23$ ) which only has a local modularity of $M O D L=0.013$. In the figures, we depict in red the edges and the vertices in the pattern extension, in gray the out-edges of the pattern (i.e., one vertex of a gray edge is contained in the pattern subgraph and the other vertex is not) and in light gray the rest of the graph

selected (left) pattern is more interesting, since it provides a more precise description. In the figures, we depict in red the edges and the vertices in the pattern subgraph; in gray, we show the out-edges of the pattern (i.e., one vertex of a gray edge is contained in the pattern extension and the other vertex is not); in light gray we depict the rest of the graph.

DBLP.C Dataset In order to show the impact of pattern selection and k-core abstraction, we first consider the local Modularities on $k$-cores with increasing $k$. For analyzing the impact of the $k$-cores we firstly consider the empty pattern, thus only focussing on the abstraction by the applied $k$-core. For the local modularity values of the empty pattern, for $k=2,3,4,5$ we observe MODL $=0.0075,0.0430,0.0915,0.1223$, respectively. Thus, we observe the clear trend that increasing $k$ yields patterns with higher connectivity structures as shown by the increasing local modularity values; similar trends are obtained for the other datasets. This complements our results in the last section, where we discussed, how increasing $k$ for the k-core abstraction together with increasing local modularity thresholds focuses on larger and more "interesting" patterns as measured by the local modularity quality function.

Figure 8 illustrates these findings: The two left graphs show examples of the $k$-cores for the empty pattern, specifically, for the 5-core with the highest local modularity, and the corresponding 3-core pattern. Areas in red indicate the core graph - both vertices and edges, blue color shows the remaining edges incident to the nodes of the core graph, while gray depicts the edges of the rest of the graph. It is easy to see that both the 3-core (2223 vertices and 9399 edges) as well as the 5-core (904 vertices and 5621 edges) demonstrate a considerably strong connectivity structure. Finally, the graph plotted on the right of Fig. 8 shows a specialization of the empty pattern on the 3-core, i.e. the pattern given by the label "mine". This pattern is obviously smaller (covering 290 vertices and 1059 edges) than the empty pattern, while its modularity structure is slightly better $(\mathrm{MODL}=0.0503)$. The 


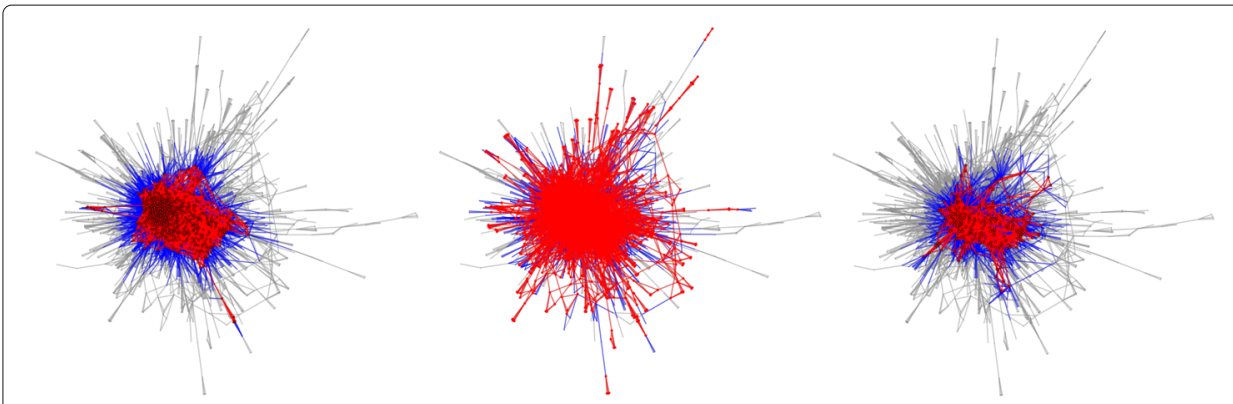

Fig. 8 Illustrative patterns (DBLP.C). Left: 5-core empty pattern with a local modularity of MODL =0.1223; middle: 3-core empty pattern with a local modularity of $\mathrm{MODL}=0.0430$; right: 3 -core "mine" pattern with a local modularity MODL $=0.0503$. In the plots, red color indicates the core graph (i.e., the in-edges of the pattern), blue color shows the edges incident to the nodes of the core graph, gray depicts the edges of the rest of the graph

left plot in Fig. 9 shows the "mine" pattern in detail, as a "zoom-in" focussing on all edges incident to nodes contained in the pattern subgraph.

Figure 9 illustrates the selection process for different 3-core patterns in detail, providing the "mine" pattern (covering 290 vertices and 1059 edges, MODL $=0.0503$ ) that is selected according to a local modularity threshold $l m=0.04$ and the "algorithm" pattern (covering 45 vertices and 93 edges, MODL $=0.0072$ ) which is a further specialization of the 3-core empty pattern. As we can clearly observe for the "mine" pattern, its structure is more interesting concerning its connectivity - i.e., its distributional unusualness compared to the expectation modeled by the null-model. This is a representative illustration, how the proposed approach using local modularity pruning achieves a better pattern selection method for the same core constraint(s).

\section{Conclusions}

In this paper, we have proposed the novel MinerLSD method for efficient local pattern mining on attributed networks. It enumerates local patterns and associated subgroups in

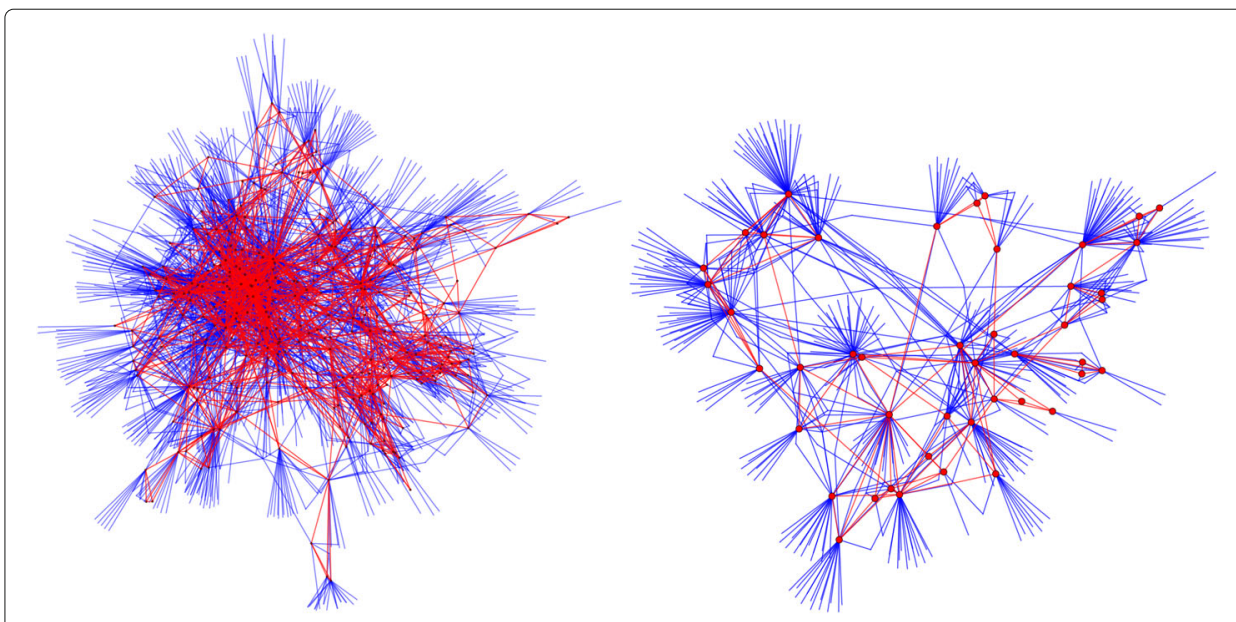

Fig. 9 Detailed view:"mine" pattern (left), with local modularity MODL $=0.0503$ vs. the lower-quality "algorithm" pattern (right), MODL $=0.0072$. In-edges (red), out-edges (blue) 
attributed networks, utilizing different pattern and graph mining techniques. In particular, MinerLSD is based on three main basic ideas: First, enumerating only closed patterns, which is particularly beneficial whenever items have dependencies. This occurs as soon as some attributes, either numeric or hierarchical, have to be translated into various items to express interesting patterns, e.g., interrelated intervals and hierarchical dependencies. Second, we focus on reducing pattern subgraphs to core subgraphs which allows both to strongly reduce the number of patterns and to focus on essential parts of graphs. Third, we select cohesive subgraphs during the search according to topological quantities as local modularity and, above all, to allow pruning by using optimistic estimates of the local modularity measure.

We performed a set of experiments in order to estimate the impact of the investigated approaches, for which we included two baseline methods, i.e., MinerLC and COMODO for comparison. The purpose was then to investigate i) the pruning efficiency of MinerLSD using the local modularity estimate as implemented in COMODO, ii) the impact of searching for closed patterns (as implemented in MinerLC) and therefore enumerating only the cohesive subgraph associated to the patterns, and iii) the added potential for pattern selection based on the combination of both k-core abstraction and local modularity selection. The latter allows to strongly reduce the number of patterns while focussing on essential parts of the graph which leads to more interesting high quality patterns. For our experiments we used a number of datasets with different characteristics, also ranging from small to large datasets in order to estimate the scalability of MinerLSD. Overall the result indicated effects that were always positive, and sometimes even crucial, for allowing to handle even rather complex and large datasets with reasonable pattern set sizes and computational effort - without using any minimum support threshold. Specifically, the results of our experiments show the efficiency of the presented method. Furthermore, we have presented exemplary results showing the benefit of pattern selection and abstraction which demonstrate the efficacy of the proposed MinerLSD approach. Overall, by implementing the different ideas. and techniques summarized above in the novel MinerLSD method, i.e., utilizing closed patterns, graph abstractions, optimistic estimate pruning using local modularity), we obtain a very flexible tool that allows to handle large graphs with adequate constraints on the subgroups and patterns to discover.

For future work, we intend to characterize the attributed graphs in terms of which pruning method is especially efficient, and to investigate other measures than local modularity in order to estimate their pruning efficiency. Furthermore, we aim to investigate other core definitions than k-cores as well. Also, focussing on sets of (local) patterns, and their relations, in order to obtain, e.g., the most diverse, representative, interesting, and relevant results, cf., (Knobbe and Ho 2006; Lemmerich et al. 2010; Van Leeuwen and Knobbe 2012; Atzmueller et al. 2015) is a further interesting research direction to consider.

\section{Endnotes}

${ }^{1}$ Available at:

http://www.stats.ox.ac.uk/ snijders/siena/s50_data.htm

${ }^{2}$ Available at:

https://www.stats.ox.ac.uk/ snijders/siena/Lazega_lawyers_data.htm

${ }^{3}$ https://grouplens.org/datasets/hetrec-2011/

${ }^{4}$ https://lipn.univ-paris13.fr/MinerLC/ 
${ }^{5}$ http://www.vikamine.org(Atzmueller and Lemmerich 2012)

${ }^{6}$ https://lipn.univ-paris13.fr/MinerLC/

\section{Acknowledgements}

Martin Atzmueller was supported in part by Université Sorbonne Paris Cité as a visiting professor.

\section{Authors' contributions}

MA and HS conceived of the idea and study, interpretation of the data and drafted the manuscript. MA and GS ran the experiments. DB and GS implemented MinerLSD and related software. All authors read and approved the final manuscript.

\section{Funding}

This work has been partially supported by the German Research Foundation (DFG) project "MODUS" (under grant AT 88/4-1). Furthermore, the research leading to these results has received funding from the Project Chistera Adalab (ANR-14-CHR2-0001-04).

\section{Availability of data and materials}

Datasets and the implementation of MinerLSD can be found at the following website: https://lipn.univ-paris13.fr/ MinerLC/

\section{Competing interests}

The authors declare that they have no competing interests.

\section{Author details}

${ }^{1}$ Tilburg University, Department of Cognitive Science and Artificial Intelligence, Warandelaan 2,5037 AB Tilburg, The Netherlands. ${ }^{2}$ Université Sorbonne Paris Cité, Paris, France. ${ }^{3}$ LIPN, Université Paris-13, SPC UMR-CNRS 7030, 93430 Villetaneuse, France. ${ }^{4}$ ISYEB UMR 7205, Museum National d'Histoire Naturelle, Paris, France.

Received: 20 December 2018 Accepted: 6 June 2019

Published online: 27 June 2019

\section{References}

Agrawal R, Srikant R (1994) Fast Algorithms for Mining Association Rules. In: Proc. VLDB. Morgan Kaufmann. pp 487-499 Almendral JA, Oliveira J, López L, Mendes J, Sanjuán MA (2007) The Network of Scientific Collaborations within the European Framework Programme. Phys A: Stat Mech Appl 384(2):675-683

Atzmueller M (2014) Data Mining on Social Interaction Networks. JDMDH 1

Atzmueller M (2015) Subgroup Discovery. WIREs DMKD 5(1):35-49

Atzmueller M (2017) Onto Explicative Data Mining: Exploratory, Interpretable and Explainable Analysis. In: Proc. Dutch-Belgian Database Day. TU Eindhoven, NL

Atzmueller M (2018) Compositional Subgroup Discovery on Attributed Social Interaction Networks. In: Proc. International Conference on Discovery Science. Springer, Berlin/Heidelberg

Atzmueller M (2019) Onto Model-based Anomalous Link Pattern Mining on Feature-Rich Social Interaction Networks. In: Proc. WWW 2019 (Companion). IW3C2 / ACM

Atzmueller M, Doerfel S, Mitzlaff F (2016) Description-Oriented Community Detection using Exhaustive Subgroup Discovery. Inf Sci 329:965-984

Atzmueller M, Lemmerich F (2009) Fast Subgroup Discovery for Continuous Target Concepts. In: Proc. 18th International Symposium on Methodologies for Intelligent Systems (ISMIS 2009), LNCS. Springer, Berlin/Heidelberg Vol. 5722. pp $1-15$

Atzmueller M, Lemmerich F (2012) VIKAMINE - Open-Source Subgroup Discovery, Pattern Mining, and Analytics. In: Proc. ECML/PKDD. Springer, Berlin/Heidelberg

Atzmueller M, Mitzlaff F (2010) Towards Mining Descriptive Community Patterns. In: Workshop on Mining Patterns and Subgroups. Lorentz Center, Leiden

Atzmueller M, Mitzlaff F (2011) Efficient Descriptive Community Mining. In: Proc. FLAIRS. AAAI Press. pp 459-464

Atzmueller M, Mueller J, Becker M (2015) Mining, Modeling and Recommending Things? in Social Media, chap. Exploratory Subgroup Analytics on Ubiquitous Data. No. 8940 in LNAI. Springer, Berlin/Heidelberg

Atzmueller M, Puppe F (2006) SD-Map - A Fast Algorithm for Exhaustive Subgroup Discovery. In: Proc. PKDD. Springer, Berlin/Heidelberg. pp 6-17

Atzmueller M, Soldano H, Santini G, Bouthinon D (2018) MinerLSD: Efficient Local Pattern Mining on Attributed Graphs. In: Proc. 2018 IEEE International Conference on Data Mining Workshops (ICDMW). IEEE Press, Boston

Balasubramanyan R, Cohen WW (2011) Block-LDA: Jointly modeling entity-annotated text and entity-entity links. In: Proc. SDM. pp 450-461

Batagelj V, Zaversnik M (2002) Generalized Cores. CoRR cs.DS/0202039

Batagelj V, Zaversnik M (2011) Fast Algorithms for Determining (Generalized) Core Groups in Social Networks. Adv Data Anal Classif 5(2):129-145

Bechara Prado A, Plantevit M, Robardet C, Boulicaut JF (2013) Mining Graph Topological Patterns: Finding Co-variations among Vertex Descriptors. IEEE Trans Knowl Data Eng 25(9):2090-2104

Belfin R, Bródka P, et al. (2018) Overlapping Community Detection using Superior Seed Set Selection in Social Networks. Comput Electr Eng 70:1074-1083

Bendimerad AA, Plantevit M, Robardet C (2016) Unsupervised Exceptional Attributed Subgraph Mining in Urban Data. In: Proc. ICDM. IEEE. pp 21-30

Boley M, Horváth T, Poigné A, Wrobel S (2010) Listing Closed Sets of Strongly Accessible Set Systems with Applications to Data Mining. TCS 411(3):691-700 
Bothorel C, Cruz JD, Magnani M, Micenkova B (2015) Clustering Attributed Graphs: Models, Measures and Methods. Netw Sci 3(03):408-444

Cantador I, Brusilovsky P, Kuflik T (2011) 2nd Workshop on Information Heterogeneity and Fusion in Recommender Systems (HetRec). In: Proc. RecSys. ACM, New York

Combe D, Largeron C, Gery M, Egyed-Zsigmond E (2015) I-louvain: An attributed graph clustering method. In: Proc. IDA. Advances in Intelligent Data Analysis. Springer, Berlin/Heidelberg. pp 181-192

Fayyad UM, Piatetsky-Shapiro G, Smyth P (1996) From Data Mining to Knowledge Discovery: An Overview. In: Fayyad UM, Piatetsky-Shapiro, Smyth P, Uthurusamy R (eds). Advances in Knowledge Discovery and Data Mining. AAAI Press, Palo Alto. pp 1-34

Fortunato S (2010) Community Detection in Graphs. Phys Rep 486(3-5):75-174

Fortunato S, Castellano C (2007) Encyclopedia of Complexity and System Science, chap. Community Structure in Graphs. Springer, Heidelberg

Freeman L (1978) Segregation In Social Networks. Sociol Methods Res 6(4):411

Galbrun E, Gionis A, Tatti N (2014) Overlapping Community Detection in Labeled Graphs. DMKD 28(5-6):1586-1610

Ganter B, Wille R (1999) Formal Concept Analysis: Mathematical Foundations. Springer Verlag, Heidelberg

Ge R, Ester M, Gao BJ, Hu Z, Bhattacharya BK, Ben-Moshe B (2008) Joint cluster analysis of attribute data and relationship data: The connected k-center problem, algorithms and applications. TKDD 2(2)

Grosskreutz H, Rüping S, Wrobel S (2008) Tight Optimistic Estimates for Fast Subgroup Discovery. In: Proc. ECML/PKDD, LNCS, vol. 5211. Springer, Berlin/Heidelberg. pp 440-456

Günnemann S, Färber I, Boden B, Seidl T (2013) GAMer: A Synthesis of Subspace Clustering and Dense Subgraph Mining. KAIS 40(2):243-278

Han J, Pei J, Yin Y (2000) Mining Frequent Patterns Without Candidate Generation. In: Proc. ACM SIGMOD. ACM Press. pp $1-12$

Kanawati R (2014) Seed-Centric Approaches for Community Detection in Complex Networks. In: International Conference on Social Computing and Social Media. Springer, Berlin/Heidelberg. pp 197-208

Kanawati R (2014) Yasca: an ensemble-based approach for community detection in complex networks. In: International Computing and Combinatorics Conference. Springer, Berlin/Heidelberg. pp 657-666

Kaytoue M, Plantevit M, Zimmermann A, Bendimerad A, Robardet C (2017) Exceptional Contextual Subgraph Mining. Mach Learn:1-41

Kibanov M, Atzmueller M, Scholz C, Stumme G (2014) Temporal Evolution of Contacts and Communities in Networks of Face-to-Face Human Interactions. Sci China Inf Sci 57(3):1-17

Klösgen W (1996) Explora: A Multipattern and Multistrategy Discovery Assistant. In: Advances in Knowledge Discovery and Data Mining. AAAI Press, Palo Alto. pp 249-271

Knobbe AJ, Cremilleux B, Fürnkranz J, Scholz M (2008) From Local Patterns to Global Models: The LeGo Approach to Data Mining. In: From Local Patterns to Global Models: Proceedings of the ECML/PKDD-08 Workshop (LeGo-08). pp 1-16

Knobbe AJ, Ho EK (2006) Pattern Teams. In: Proc. PKDD. Springer, Berlin/Heidelberg. pp 577-584

Kumar R, Novak J, Tomkins A (2006) Structure and Evolution of Online Social Networks. In: Proc. ACM SIGKDD. ACM. pp 611-617

Lancichinetti A, Fortunato S, Kertész J (2009) Detecting the Overlapping and Hierarchical Community Structure in Complex Networks. New J Phys $11(3)$

Lancichinetti A, Kivelä M, Saramäki J, Fortunato S (2010) Characterizing the community structure of complex networks. PloS One 5(8):e11,976

Lazega E (2001) The Collegial Phenomenon: The Social Mechanisms of Cooperation Among Peers in a Corporate Law Partnership. Oxford University Press

Lemmerich F, Atzmueller M, Puppe F (2016) Fast Exhaustive Subgroup Discovery with Numerical Target Concepts. Data Min Knowl Discov 30:711-762

Lemmerich F, Becker M, Atzmueller M (2012) Generic Pattern Trees for Exhaustive Exceptional Model Mining. In: Proc. ECML PKDD, LNCS, vol. 7524. Springer, Berlin/Heidelberg. pp 277-292

Lemmerich F, Rohlfs M, Atzmueller M (2010) Fast Discovery of Relevant Subgroup Patterns. In: Proc. FLAIRS. AAAI Press, Palo Alto. pp 428-433

Leskovec J, Lang KJ, Dasgupta A, Mahoney MW (2008) Community Structure in Large Networks: Natural Cluster Sizes and the Absence of Large Well-Defined Clusters. CoRR. abs/0810.1355

Leskovec J, Lang KJ, Mahoney M (2010) Empirical Comparison of Algorithms for Network Community Detection. In: Proc. WWW. ACM, New York. pp 631-640

Mitzlaff F, Atzmueller M, Benz D, Hotho A, Stumme G (2011) Community Assessment using Evidence Networks. In: Analysis of Social Media and Ubiquitous Data, LNAl, vol. 6904. Springer, Berlin/Heidelberg

Mitzlaff F, Atzmueller M, Hotho A, Stumme G (2014) The Social Distributional Hypothesis. SNAM 4(216)

Mitzlaff F, Atzmueller M, Stumme G, Hotho A (2013) Semantics of User Interaction in Social Media. In: Complex Networks $\mathrm{IV}, \mathrm{SCl}$, vol. 476. Springer, Berlin/Heidelberg

Morik K (2002) Detecting Interesting Instances. In: Hand D, Adams N, Bolton R (eds). Pattern Detection and Discovery, LNCS, vol. 2447. Springer, Berlin/Heidelberg. pp 13-23

Morik K, Boulicaut J, Siebes A (2005) Local Pattern Detection, International Seminar, Dagstuhl Castle, Germany, April 12-16, 2004, Revised Selected Papers, LNCS, vol. 3539. Springer, Berlin/Heidelberg

Moser F, Colak R, Rafiey A, Ester M (2009) Mining Cohesive Patterns from Graphs with Feature Vectors. In: Proc. SDM. pp 593-604

Newman ME, Girvan M (2004) Finding and Evaluating Community Structure in Networks. Phys Rev E Stat Nonlin Soft Matter Phys 69(2):1-15

Newman MEJ (2003) The Structure and Function of Complex Networks. SIAM Rev 45(2):167-256

Newman MEJ (2004) Detecting Community Structure in Networks. In: EPJ 38

Newman MEJ (2006) Modularity and Community Structure in Networks. PNAS 103(23):8577-8582

Nicolle R, Radvanyi F, Elati M (2015) Coregnet: Reconstruction and Integrated Analysis of Co-Regulatory Networks. Bioinformatics 
Nicosia V, Mangioni G, Carchiolo V, Malgeri M (2009) Extending the Definition of Modularity to Directed Graphs with Overlapping Communities. J Stat Mech

Palla G, Derenyi I, Farkas I, Vicsek T (2005) Uncovering the Overlapping Community Structure of Complex Networks in Nature and Society. Nature 435(7043):814-818

Palla G, Farkas IJ, Pollner P, Derenyi I, Vicsek T (2007) Directed Network Modules. New J Phys 9(6):186

Pasquier N, Bastide Y, Taouil R, Lakhal L (1999) Efficient Mining of Association Rules using Closed Itemset Lattices. Inf Syst 24(1):25-46

Peng C, Kolda TG, Pinar A (2014) Accelerating Community Detection by Using k-core Subgraphs 1403:2226. arXiv preprint arXiv

Pernelle N, Rousset MC, Soldano H, Ventos V (2002) ZooM: A Nested Galois Lattices-Based System for Conceptual Clustering. J Exp Theor Artif Intell 2/3(14):157-187

Pool S, Bonchi F, van Leeuwen M (2014) Description-driven Community Detection. ACM Trans Intell Syst Technol 5(2)

Seidman SB (1983) Network Structure and Minimum Degree. Soc Netw 5:269-287

Silva A, Meira Jr. W, Zaki MJ (2012) Mining attribute-structure correlated patterns in large attributed graphs. Proc VLDB Endow 5(5):466-477

Silva A, Meira W, Zaki MJ (2012) Mining Attribute-Structure Correlated Patterns in Large Attributed Graphs. Proc VLDB Endowment 5(5):466-477

Smith LM, Zhu L, Lerman K, Percus AG (2014) Partitioning networks with node attributes by compressing information flow. CoRR. abs/1405.4332

Soldano H, Santini G (2014) Graph Abstraction for Closed Pattern Mining in Attributed Networks. In: Proc. ECAl, FAIA, vol. 263. IOS Press. pp 849-854

Soldano H, Santini G, Bouthinon D (2015) Local Knowledge Discovery in Attributed Graphs. In: Proc. ICTAI. IEEE. pp 250-257

Soldano H, Santini G, Bouthinon D, Lazega E (2017) Hub-Authority Cores and Attributed Directed Network Mining. In: Proc. ICTAI. IEEE, Boston, MA

Steinhaeuser K, Chawla NV (2008) Community detection in a large real-world social network. In: Social computing, behavioral modeling, and prediction. Springer. pp 168-175

Uno T, Asai T, Uchida Y, Arimura H (2004) An Efficient Algorithm for Enumerating Closed Patterns in Transaction Databases. In: Proc. Discovery Science. pp 16-31

Van Leeuwen M, Knobbe A (2012) Diverse Subgroup Set Discovery. Data Min Knowl Discov 25(2):208-242

Wasserman S, Faust K (1994) Social Network Analysis: Methods and Applications, 1 edn. No. 8 in Structural analysis in the social sciences. Cambridge University Press

Wille R (1982) Restructuring Lattice Theory. In: Symposium on Ordered Sets. University of Calgary, Boston. pp 445-470

Wrobel S (1997) An Algorithm for Multi-Relational Discovery of Subgroups. In: Proc. PKDD. Springer, Berlin/Heidelberg. pp 78-87

Xie J, Kelley S, Szymanski BK (2013) Overlapping Community Detection in Networks: The State-of-the-art and Comparative Study. ACM Comput Surv 45(4):43:1-43:35

Xie J, Szymanski BK (2013) LabelRank: A Stabilized Label Propagation Algorithm for Community Detection in Networks. In: Proc. IEEE Network Science Workshop, West Point

Xu Z, Ke Y, Wang Y, Cheng H, Cheng J (2012) A model-based approach to attributed graph clustering. In: Proc. SIGMOD. pp 505-516

Yakoubi Z, Kanawati R (2014) Licod: Leader-driven approaches for community detection. Vietnam J Comput Sci 1(4):241-256

Yang J, Leskovec J (2012) Defining and Evaluating Network Communities Based on Ground-truth. In: Proc. ACM SIGKDD Workshop on Mining Data Semantics, MDS '12. ACM, New York. pp 3:1-3:8

Zhou Y, Cheng H, Yu JX (2009) Graph clustering based on structural/attribute similarities. PVLDB 2(1):718-729

Zhu L, Ng WK, Cheng J (2011) Structure and attribute index for approximate graph matching in large graphs. Inf Syst 36(6):958-972

Publisher's Note

Springer Nature remains neutral with regard to jurisdictional claims in published maps and institutional affiliations.

\section{Submit your manuscript to a SpringerOpen ${ }^{0}$ journal and benefit from:}

- Convenient online submission

- Rigorous peer review

- Open access: articles freely available online

- High visibility within the field

- Retaining the copyright to your article

Submit your next manuscript at $\boldsymbol{\triangleright}$ springeropen.com 